\title{
Total Synthesis of the Proposed Microcyclamides MZ602 and MZ568
}

Yi Liu,,${ }^{\dagger}$ Xiangyun Zhao, ${ }^{\dagger}$ Hongbo Wang, ${ }^{\ddagger}$ Huili Liu, ${ }^{\ddagger}$ Zhuyin Sui, ${ }^{\dagger}$ Bingfei, Yan, ${ }^{\dagger}$ Yuguo $\mathrm{Du}^{*}$

${ }^{\dagger}$ School of Chemistry and Chemical Engineering, Yantai University, Yantai 264005, China;

${ }^{\S}$ State Key Laboratory of Environmental Chemistry and Eco-toxicology, Research Center for Eco-Environmental Sciences, Chinese Academy of Sciences, Beijing 100085, China;

‡ Key Laboratory of Molecular Pharmacology and Drug Evaluation (Yantai University), Ministry of Education, Yantai University, Yantai 264005, China. 


\section{Contents}

1. Comparison of Spectroscopic Data of Our Synthetic Natural Products with Isolation Reports ...............................................................................................................................S3

2. X-ray Crystallography of Compound 36 ...................................................................S10

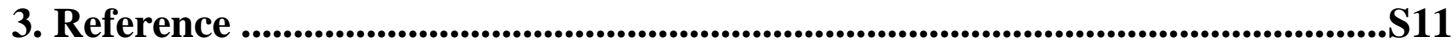

4. The Data of Control Experiments ..........................................................................S12

5. Copies of NMR Spectra of Synthesized Compounds 1 4, 11, 12, 16 21, 23, 28, 29, 31 34, 36 S12 


\section{Comparison of Spectroscopic Data of Natural products ${ }^{1}$ and Synthetic Compounds.}

Table S1. Comparison of the Spectroscopic Data of Natural Microcyclamide MZ602 $^{1}$ and Synthetic Compound 1

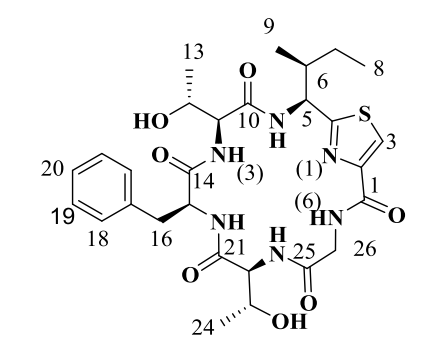

\begin{tabular}{|c|c|c|c|c|c|c|}
\hline \multicolumn{7}{|c|}{ Microcyclamides MZ602 } \\
\hline \multirow{2}{*}{ Position } & \multicolumn{3}{|c|}{ Microcyclamide $\mathrm{MZ602}^{\mathrm{a}}$} & \multicolumn{3}{|c|}{ Synthetic Compound $\mathbf{1}^{\mathrm{b}}$} \\
\hline & $\delta_{\mathrm{H}},\left(\mathrm{M},{ }^{\mathrm{c}} J^{\mathrm{d}}\right)$ & $\delta_{\mathrm{C}}$ & HMBC & $\delta_{\mathrm{H}},\left(\mathrm{M},{ }^{\mathrm{c}} J^{\mathrm{d}}\right)$ & $\delta_{\mathrm{C}}$ & HMBC \\
\hline 1 & & 161.1 & $3,26, \mathrm{~N}(6) \mathrm{H}$ & & 161.1 & $26, \mathrm{~N}(6) \mathrm{H}$ \\
\hline 2 & & 148.8 & 3 & & 148.8 & 3 \\
\hline 3 & $8.22(\mathrm{~s})$ & 124.0 & & $8.22(\mathrm{~s})$ & 124.0 & \\
\hline 4 & & 168.8 & 3,5 & & 168.8 & 3,5 \\
\hline 5 & $5.16(\mathrm{dd}, 8.8,5.7)^{\mathrm{e}}$ & 55.1 & $8,9, \mathrm{~N}(2) \mathrm{H}$ & $5.11(\mathrm{dd}, 8.4,5.6)$ & 55.1 & $8,9, \mathrm{~N}(2) \mathrm{H}$ \\
\hline 6 & $2.00(\mathrm{~m})$ & 39.2 & $5,7,7,8,9$ & $2.00(\mathrm{~m})$ & 39.2 & $5,8,9$ \\
\hline 7 & $1.41(\mathrm{~m}), 1.08(\mathrm{~m})$ & 24.6 & $5,8,9$ & $1.41(\mathrm{~m}), 1.08(\mathrm{~m})$ & 24.6 & $5,8,9$ \\
\hline 8 & $0.86(t, 7.7)$ & 11.8 & 7,7 & $0.86(t, 7.2)$ & 11.8 & 7,7 \\
\hline 9 & $0.87(\mathrm{~d}, 7.0)$ & 15.7 & $5,7,7$ & $0.87(\mathrm{~d}, 6.8)$ & 15.7 & 5 \\
\hline $\mathrm{N}(2) \mathrm{H}$ & $7.74(\mathrm{~d}, 8.2)$ & & & $7.74(\mathrm{~d}, 8.8)$ & & \\
\hline 10 & & 169.9 & $11, \mathrm{~N}(2) \mathrm{H}$ & & 169.9 & $\mathrm{~N}(2) \mathrm{H}$ \\
\hline
\end{tabular}

S3 


\begin{tabular}{|c|c|c|c|c|c|c|}
\hline 11 & $3.95(\mathrm{~m})$ & 61.0 & $13, \mathrm{~N}(3) \mathrm{H}$ & $3.95(\mathrm{~m})$ & 61.0 & $13, \mathrm{~N}(3) \mathrm{H}$ \\
\hline 12 & $4.16(\mathrm{~m})$ & 65.3 & $11,13, \mathrm{~N}(3) \mathrm{H}$ & $4.16(\mathrm{~m})$ & 65.3 & 11,13 \\
\hline 13 & $1.09(\mathrm{~d}, 6.2)$ & 20.5 & 12 & $1.09(\mathrm{~d}, 6.4)$ & 20.5 & \\
\hline $\mathrm{N}(3) \mathrm{H}$ & $8.15(\mathrm{~d}, 7.0)$ & & & $8.15(\mathrm{~d}, 7.2)$ & & \\
\hline $12-\mathrm{OH}$ & $5.16($ br s) & & & 5.18 (br s) & & \\
\hline 14 & & 172.0 & $\begin{array}{c}11, \mathrm{~N}(3) \mathrm{H}, 15 \\
16\end{array}$ & & 172.0 & $\mathrm{~N}(3) \mathrm{H}$ \\
\hline 15 & $4.49(\mathrm{~m})$ & 54.4 & $16, \mathrm{~N}(4) \mathrm{H}$ & $4.49(\mathrm{~m})$ & 54.4 & 16 \\
\hline 16 & $\begin{array}{c}3.32(\mathrm{~m}), 2.87(\mathrm{dd}, \\
14.5,11.4)\end{array}$ & 37.2 & $15,18,18$ & $\begin{array}{c}3.32(\mathrm{~m}), 2.87(\mathrm{dd} \\
14.0,10.8)\end{array}$ & 37.2 & $18,18^{\prime}$ \\
\hline 17 & & 138.0 & $\begin{array}{c}16,16^{\prime}, 19, \\
19^{\prime}\end{array}$ & & 138.0 & $16,19,19^{\prime}$ \\
\hline $18,18^{\prime}$ & $7.27(\mathrm{~m})$ & 129.2 & 16,20 & $7.27(\mathrm{~m})$ & 129.2 & 16,20 \\
\hline $19,19^{\prime}$ & $7.24(\mathrm{~m})$ & 128.2 & & $7.24(\mathrm{~m})$ & 128.2 & \\
\hline 20 & $7.17(\mathrm{~m})$ & 126.4 & $\begin{array}{c}18,18,, 19, \\
19,\end{array}$ & $7.17(\mathrm{~m})$ & 126.4 & $\begin{array}{c}18,18^{\prime}, 19, \\
19\end{array}$ \\
\hline $\mathrm{N}(4) \mathrm{H}$ & $8.09(\mathrm{~d}, 7.9)$ & & & $8.09(\mathrm{~d}, 7.6)$ & & \\
\hline 21 & & 170.6 & $\mathrm{~N}(4) \mathrm{H}, 23$ & & 170.6 & $\mathrm{~N}(4) \mathrm{H}, 23$ \\
\hline 22 & $3.90(\mathrm{~m})$ & 60.7 & $24, \mathrm{~N}(5) \mathrm{H}$ & $3.90(\mathrm{~m})$ & 60.7 & 24 \\
\hline 23 & $3.86(\mathrm{~m})$ & 66.1 & 22,24 & $3.86(\mathrm{~m})$ & 66.1 & 22,24 \\
\hline 24 & $0.82(\mathrm{~d}, 6.1)$ & 20.2 & 23 & $0.82(\mathrm{~d}, 6.0)$ & 20.2 & 23 \\
\hline $\mathrm{N}(5) \mathrm{H}$ & $8.09(\mathrm{~d}, 7.9)^{\mathrm{e}}$ & & & $7.83(\mathrm{~d}, 7.6)$ & & \\
\hline $23-\mathrm{OH}$ & 4.84 (br s) & & & 4.84 (br s) & & \\
\hline 25 & & 169.1 & $\mathrm{~N}(5) \mathrm{H}, 26,26^{\prime}$ & & 169.1 & $\begin{array}{c}\mathrm{N}(5) \mathrm{H}, 26, \\
26\end{array}$ \\
\hline $26.26^{\prime}$ & $3.94(\mathrm{~m})$ & 43.6 & $\mathrm{~N}(5) \mathrm{H}$ & $3.94(\mathrm{~m})$ & 43.6 & \\
\hline
\end{tabular}


${ }^{\mathrm{a}} 500 \mathrm{~Hz}$ for ${ }^{1} \mathrm{H}$ and $125 \mathrm{~Hz}$ for ${ }^{13} \mathrm{C} ;{ }^{\mathrm{b}} 400 \mathrm{~Hz}$ for ${ }^{1} \mathrm{H}$ and $100 \mathrm{~Hz}$ for ${ }^{13} \mathrm{C} ;{ }^{\mathrm{c}} \mathrm{M}=$ multiplicity; ${ }^{\mathrm{d}} \mathrm{J}$ in $\mathrm{Hz} ;{ }^{\mathrm{e}}$ reported data not match with original spectrum. 
Table S2. Comparison of the Spectroscopic Data of Natural Microcyclamide MZ568 ${ }^{1}$ and Synthetic Compound 2

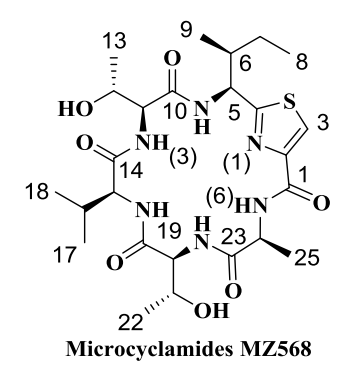

\begin{tabular}{|c|c|c|c|c|c|c|}
\hline \multirow{2}{*}{ Position } & \multicolumn{3}{|c|}{ Microcyclamide MZ568 ${ }^{\mathrm{a}}$} & \multicolumn{3}{|c|}{ Synthetic Compound $2^{\mathrm{b}}$} \\
\hline & $\delta_{\mathrm{H}},\left(\mathrm{M},{ }^{\mathrm{c}} J^{\mathrm{d}}\right)$ & $\delta_{\mathrm{C}},(\mathrm{M})^{\mathrm{c}}$ & HMBC & $\delta_{\mathrm{H}},\left(\mathrm{M},{ }^{\mathrm{c}} J^{\mathrm{d}}\right)$ & $\delta_{\mathrm{C}},(\mathrm{M})^{\mathrm{c}}$ & HMBC \\
\hline 1 & & 160.7 & $\mathrm{~N}(6) \mathrm{H}$ & & 161.2 & $\mathrm{~N}(6) \mathrm{H}$ \\
\hline 2 & & 148.4 & 3 & & 150.1 & 3 \\
\hline 3 & $8.24(\mathrm{~s})$ & 124.9 & & $8.17(\mathrm{~s})$ & 123.5 & \\
\hline 4 & & 169.9 & $3,5, \mathrm{~N}(2) \mathrm{H}$ & & 167.8 & 3,5 \\
\hline 5 & $5.09(\mathrm{dd}, 8.5,4.5)$ & 55.7 & 8,9 & $5.02(\mathrm{~s})$ & 53.8 & 9 \\
\hline 6 & $2.03(\mathrm{~m})$ & 38.9 & 8,9 & $2.14(\mathrm{~m})$ & 38.4 & $5,7,8,9$ \\
\hline 7 & $1.29(\mathrm{~m}), 1.05(\mathrm{~m})$ & 23.9 & 8,9 & $1.54(\mathrm{~m}), 1.11(\mathrm{~m})$ & 24.8 & 8,9 \\
\hline 8 & $0.87(\mathrm{t}, 7.3)$ & 11.7 & & $0.86(\mathrm{t}, 7.6)$ & 10.9 & 7 \\
\hline 9 & $0.91(\mathrm{~d}, 6.6)$ & 16.0 & 8 & $0.93(\mathrm{~d}, 6.8)$ & 15.4 & $5,6,7$ \\
\hline $\mathrm{N}(2) \mathrm{H}$ & $7.19(\mathrm{~d}, 8.6)$ & & & $7.59(\mathrm{~d}, 7.6)$ & & \\
\hline 10 & & 170.3 & $11, \mathrm{~N}(2) \mathrm{H}$ & & 169.6 & $\mathrm{~N}(2) \mathrm{H}, 11$ \\
\hline 11 & $3.88(t, 4.5)$ & 61.9 & 13 & $3.79(\mathrm{dd}, 7.6,5.6)$ & 57.6 & 13 \\
\hline 12 & $4.14(\mathrm{ddq}, 4.5,5.6,6.6)$ & 65.1 & 13 & $4.30(\mathrm{~d}, 3.2)$ & 65.2 & 13 \\
\hline 13 & $1.17(\mathrm{~d}, 6.6)$ & 20.8 & & $1.13(\mathrm{~d}, 6.0)$ & 21.0 & \\
\hline
\end{tabular}




\begin{tabular}{|c|c|c|c|c|c|c|}
\hline $\mathrm{N}(3) \mathrm{H}$ & $8.12(\mathrm{~d}, 4.8)$ & & & $7.94(\mathrm{~d}, 5.6)$ & & \\
\hline $12-\mathrm{OH}$ & $4.96(d, 5.6)$ & & & $5.04(\mathrm{~d}, 3.2)$ & & \\
\hline 14 & & 172.5 & $\mathrm{~N}(3) \mathrm{H}, 15$ & & 170.8 & $\mathrm{~N}(3) \mathrm{H}, 15$ \\
\hline 15 & $4.44(\mathrm{dd}, 9.6,2.2)$ & 55.9 & 17,18 & $4.57(\mathrm{dd}, 10.0,7.2)$ & 59.9 & 17,18 \\
\hline 16 & $2.29(\mathrm{~m})$ & 31.0 & 17,18 & $2.14(\mathrm{~m})$ & 29.6 & 17,18 \\
\hline 17 & $0.84(\mathrm{~d}, 6.6)$ & 20.0 & 18 & $0.93(\mathrm{~d}, 6.8)$ & 19.1 & 18 \\
\hline 18 & $0.48(\mathrm{~d}, 6.7)$ & 15.6 & 17 & $0.79(\mathrm{~d}, 6.8)$ & 17.8 & 17 \\
\hline $\mathrm{N}(4) \mathrm{H}$ & $6.73(\mathrm{~d}, 9.6)$ & & & $7.59(\mathrm{~d}, 7.6)$ & & \\
\hline 19 & & 170.3 & $\mathrm{~N}(4) \mathrm{H}, 20$ & & 171.2 & $\mathrm{~N}(4) \mathrm{H}, 20$ \\
\hline 20 & $3.96(\mathrm{dd}, 7.7,3.8)$ & 61.2 & 22 & $3.84(t, 5.2)$ & 62.0 & 22 \\
\hline 21 & $4.11(\mathrm{~m})$ & 65.5 & 22 & $4.27(\mathrm{~d}, 2.8)$ & 67.8 & 22 \\
\hline 22 & $1.14(\mathrm{~d}, 6.4)$ & 20.8 & & $1.08(\mathrm{~d}, 6.4)$ & 19.9 & \\
\hline $\mathrm{N}(5) \mathrm{H}$ & $8.45(\mathrm{~d}, 7.6)$ & & & $7.97(\mathrm{~d}, 9.2)$ & & \\
\hline $21-\mathrm{OH}$ & $4.79(\mathrm{~d}, 6.1)$ & & & $4.99(\mathrm{~m})$ & & \\
\hline 23 & & 171.2 & $\mathrm{~N}(5) \mathrm{H}, 24,25$ & & 171.9 & $\mathrm{~N}(5) \mathrm{H}, 24,25$ \\
\hline 24 & $4.82(\mathrm{~m})$ & 48.4 & 25 & $4.15(\mathrm{q}, 6.0)$ & 49.9 & 25 \\
\hline 25 & $1.22(\mathrm{~d}, 6.6)$ & 16.8 & & $1.45(\mathrm{~d}, 7.2)$ & 17.6 & \\
\hline $\mathrm{N}(6) \mathrm{H}$ & $8.53(\mathrm{~d}, 7.9)$ & & & $8.50(\mathrm{~d}, 10.0)$ & & \\
\hline
\end{tabular}

${ }^{\mathrm{a}} 400 \mathrm{~Hz}$ for ${ }^{1} \mathrm{H}$ and $100 \mathrm{~Hz}$ for ${ }^{13} \mathrm{C} ;{ }^{\mathrm{b}} 400 \mathrm{~Hz}$ for ${ }^{1} \mathrm{H}$ and $100 \mathrm{~Hz}$ for ${ }^{13} \mathrm{C} ;{ }^{\mathrm{c}} \mathrm{M}=$ multiplicity; ${ }^{\mathrm{d}} \mathrm{J}$ in $\mathrm{Hz}$. 


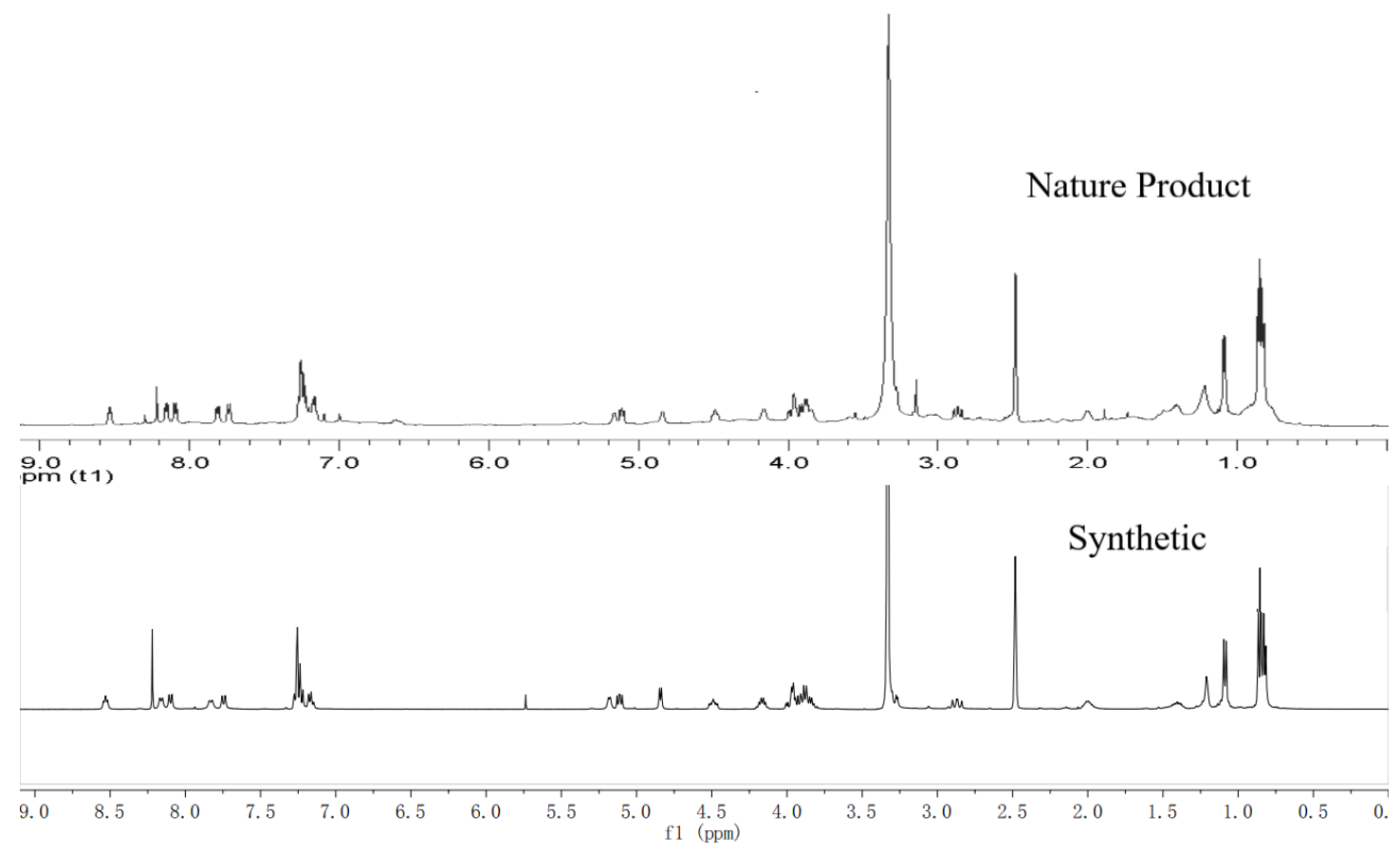

Figure S1: ${ }^{1}$ H NMR Spectroscopic Data of Natural Microcyclamide MZ602 and Synthetic 1 in ${ }_{\text {DMSO-d }}$
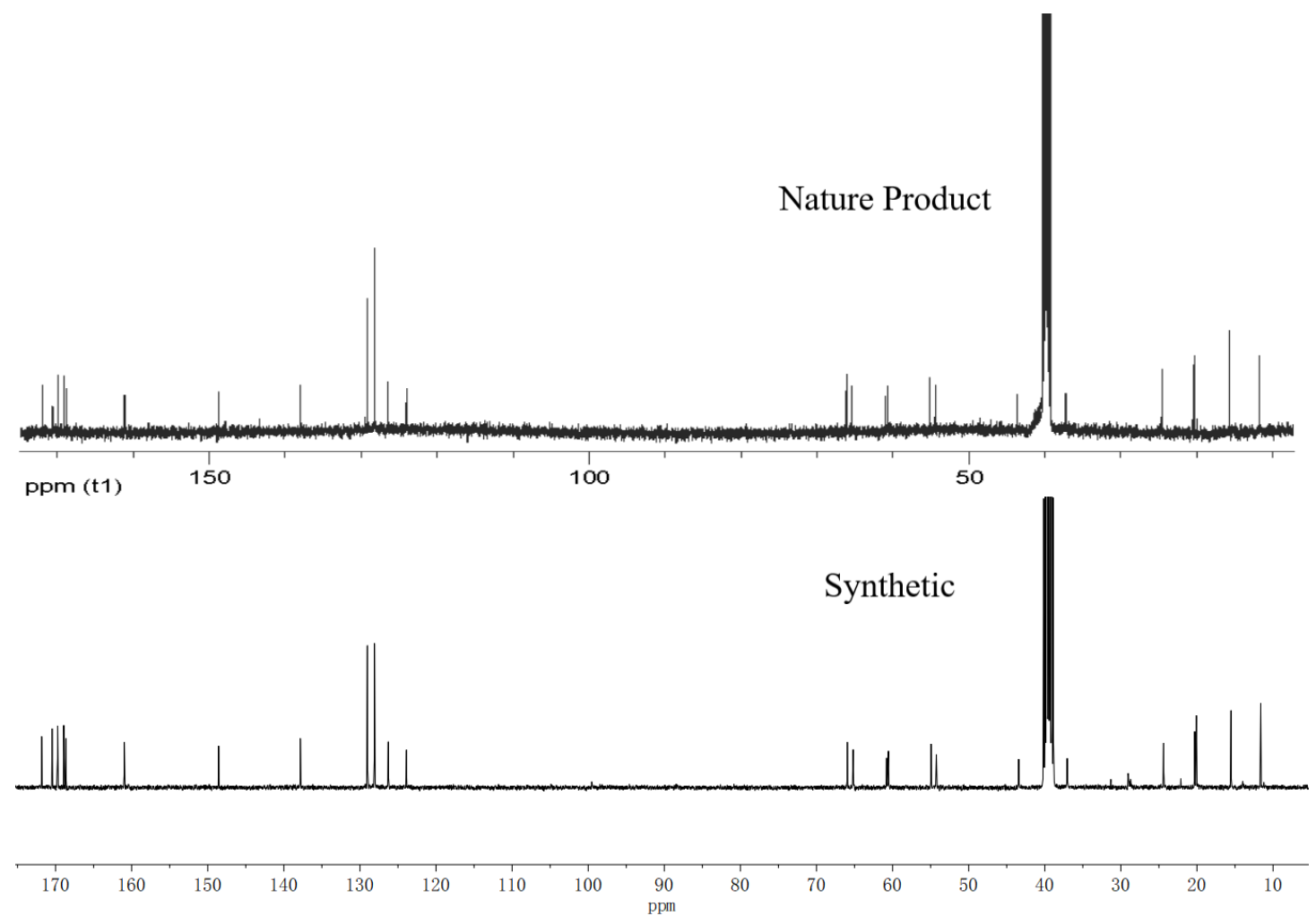

Figure S2: ${ }^{13} \mathrm{C}$ NMR Spectroscopic Data of Natural Microcyclamide MZ602 and Synthetic 1 in DMSO-d $_{6}$ 


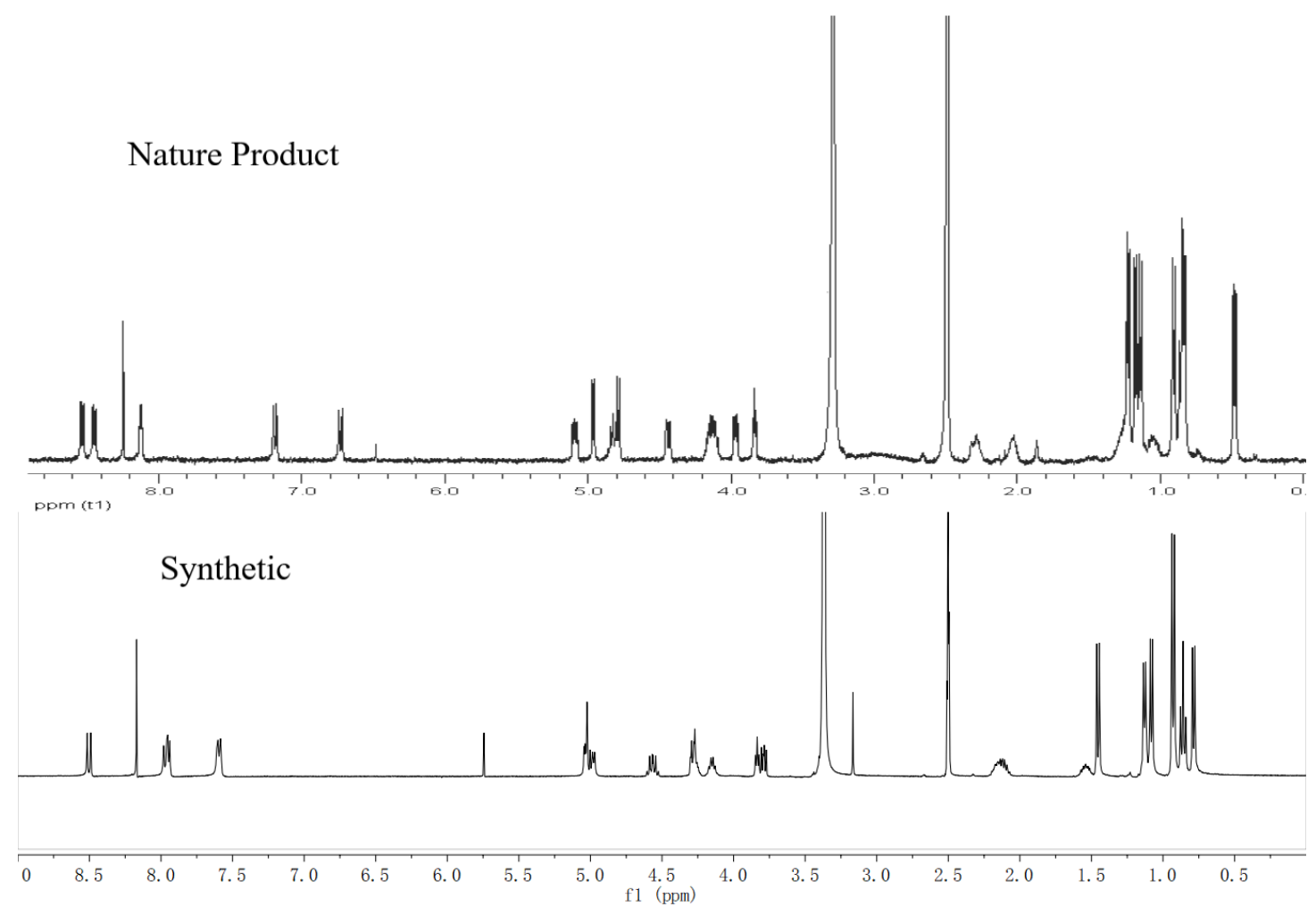

Figure S3: ${ }^{1} \mathrm{H}$ NMR Spectroscopic Data of Natural Microcyclamide MZ568 and Synthetic 2 in ${ }^{\text {DMSO-d }} 6$
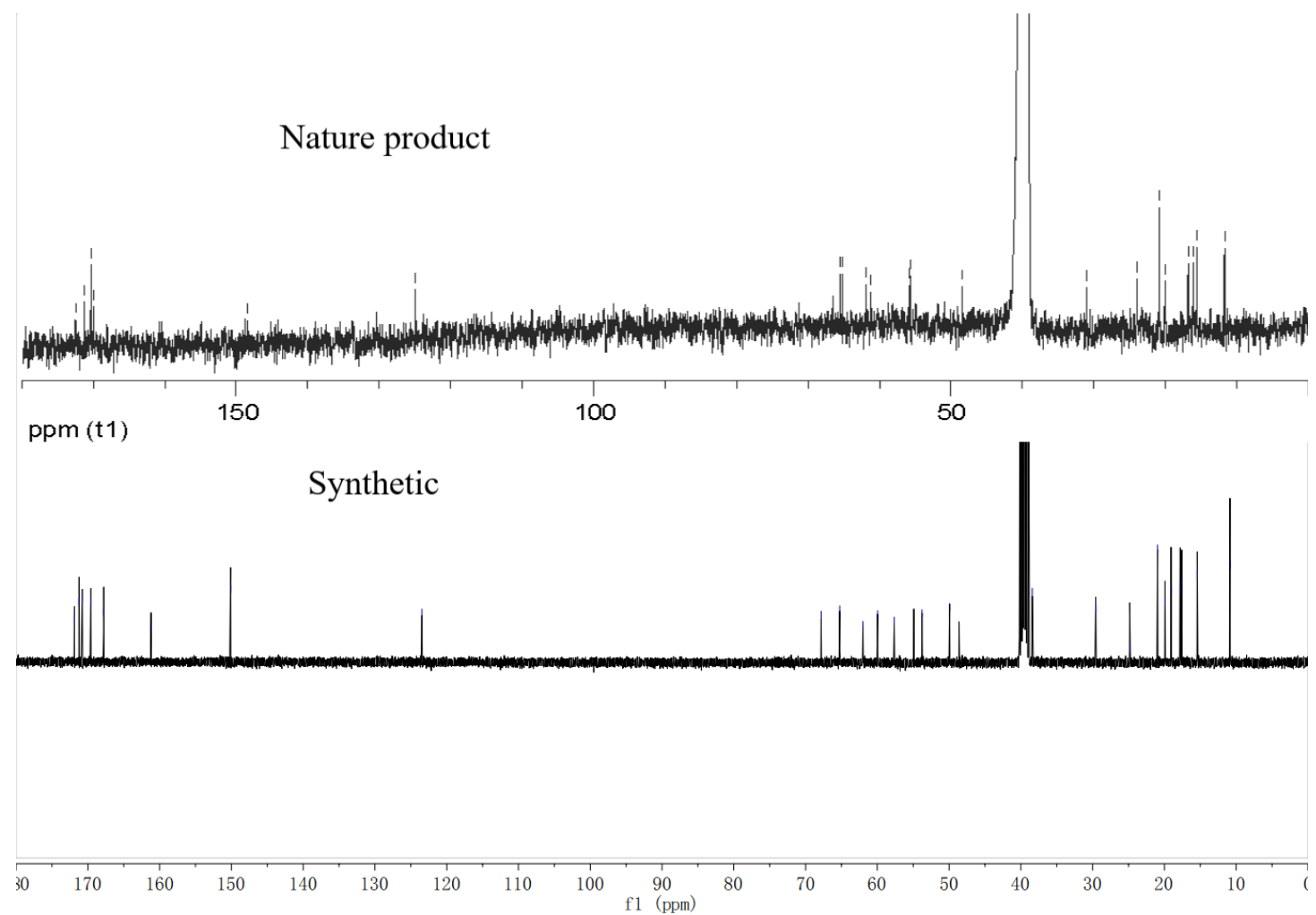

Figure S4: ${ }^{13} \mathrm{C}$ NMR Spectroscopic Data of Natural Microcyclamide MZ568 and Synthetic 2 in DMSO-d $_{6}$ 


\section{X-ray Crystallography of Compound 36}
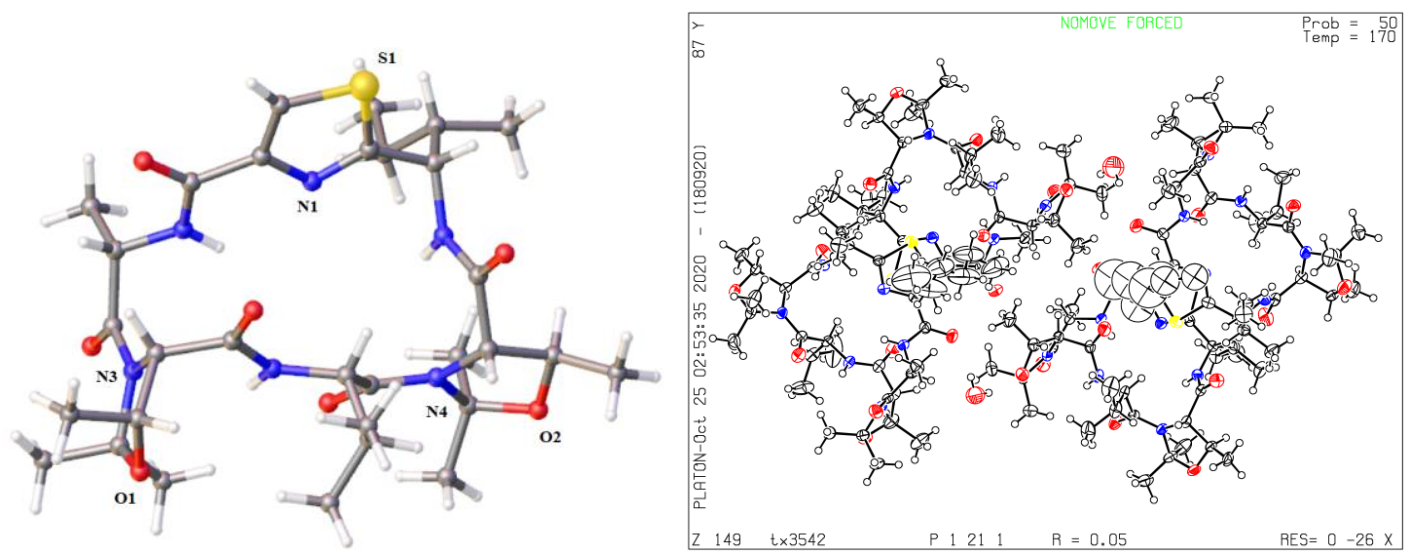

Figure S5. The Crystal Structure of $\mathbf{3 6}$ with Thermal Ellipsoid Contour at 50\% Probability Level.

Single crystals of compound 36 (Identification code tx3452_sq) was obtained by slow solvent evaporation from hexane and ethyl acetate solution $(\mathrm{v} / \mathrm{v}=1 / 1)$ over the course of two days. The single crystal X-ray diffraction studies were carried out on a XtaLAB Synergy R, HyPix diffractometer. The crystal was kept at $169.97(14) \mathrm{K}$ during data collection. Using Olex2 $2^{2}$, the structure was solved with the ShelXT ${ }^{3}$ structure solution program using Intrinsic Phasing and refined with the ShelXL ${ }^{4}$ refinement package using Least Squares minimisation. Solution by direct methods produced a complete phasing model consistent with the proposed structure. All nonhydrogen atoms were refined anisotropically by full-matrix least-squares (SHELXL-2014). All carbon bonded hydrogen atoms were placed using a riding model. Their positions were constrained relative to their parent atom using the appropriate HFIX command in SHELXL-2014. All other hydrogen atoms (H-bonding) were located in the difference map. Their relative positions were restrained using DFIX commands and their thermals freely refined. The absolute stereochemistry of the molecule was established by anomalous dispersion using the Parson's method with a Flack parameter of 0.041(43). 
Table S3. Crystal Data and Structure Refinement for 36.

\begin{tabular}{|c|c|}
\hline Identification code & tx3542_sq \\
\hline Empirical formula & $\mathrm{C}_{130} \mathrm{H}_{210} \mathrm{~N}_{24} \mathrm{O}_{30} \mathrm{~S}_{4}$ \\
\hline Formula weight & 2717.45 \\
\hline Temperature/K & $169.97(14)$ \\
\hline Crystal system & monoclinic \\
\hline Space group & $P 2_{1}$ \\
\hline$a / \AA$ & $14.92610(10)$ \\
\hline$b / \AA$ & $22.70870(10)$ \\
\hline$c / \AA$ & $22.59850(10)$ \\
\hline$\alpha /^{\circ}$ & 90 \\
\hline$\beta /^{\circ}$ & $92.3510(10)$ \\
\hline$\gamma /{ }^{\circ}$ & 90 \\
\hline Volume $/ \AA^{3}$ & 7653.37(7) \\
\hline $\mathrm{Z}$ & 2 \\
\hline$\rho_{\text {calc }} \mathrm{g} / \mathrm{cm}^{3}$ & 1.179 \\
\hline$\mu / \mathrm{mm}^{-1}$ & 1.175 \\
\hline $\mathrm{F}(000)$ & 2924.0 \\
\hline Crystal size $/ \mathrm{mm}^{3}$ & $0.5 \times 0.3 \times 0.15$ \\
\hline Radiation & $\mathrm{CuK} \alpha(\lambda=1.54184)$ \\
\hline $2 \Theta$ range for data collection ${ }^{\circ}$ & 5.52 to 151.212 \\
\hline Index ranges & $-18 \leq \mathrm{h} \leq 18,-28 \leq \mathrm{k} \leq 28,-26 \leq 1 \leq 28$ \\
\hline Reflections collected & 112031 \\
\hline Independent reflections & $30544\left[R_{\text {int }}=0.0340, R_{\text {sigma }}=0.0304\right]$ \\
\hline Data/restraints/parameters & $30544 / 1 / 1745$ \\
\hline Goodness-of-fit on $\mathrm{F}^{2}$ & 1.040 \\
\hline Final $R$ indexes $[\mathrm{I}>=2 \sigma(\mathrm{I})]$ & $\mathrm{R}_{1}=0.0483, \mathrm{wR}_{2}=0.1360$ \\
\hline Final $\mathrm{R}$ indexes [all data] & $\mathrm{R}_{1}=0.0493, \mathrm{wR}_{2}=0.1368$ \\
\hline Largest diff. peak/hole / e $\AA^{-3}$ & $0.65 /-0.48$ \\
\hline Flack parameter & $0.013(3)$ \\
\hline
\end{tabular}

\section{Reference}

[1] Zafrir, E.; Carmeli, S. Two New Microcyclamides from a Water Bloom of the Cyanobacterium Microcystis sp. Tetrahedron Lett., 2010, 51, 6602-6604.

[2] Dolomanov, O.V., Bourhis, L.J., Gildea, R.J, Howard, J.A.K. \& Puschmann, H. J. Appl. Cryst. 2009, 42, 339-341.

[3]Sheldrick, G.M. Acta Cryst. A 2015, 71, 3-8.

[4]Sheldrick, G.M. Acta Cryst. C 2015, 71, 3-8. 


\section{The Data of Control Experiments}

\subsection{Boc-L-Ile-Thz-OAllyl (5)}
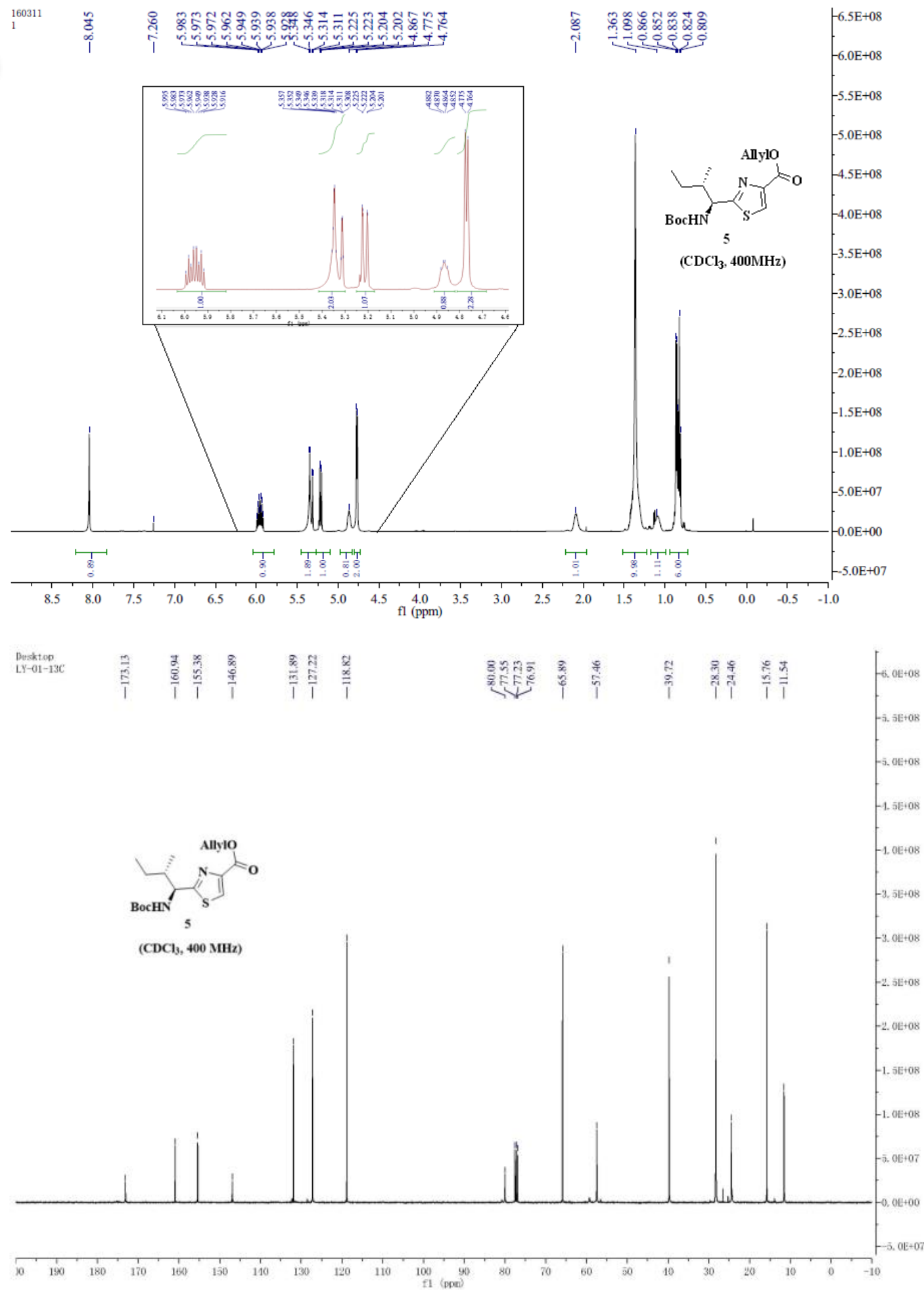

Figure S6: ${ }^{1} \mathrm{H}$ NMR and ${ }^{13} \mathrm{C}$ NMR Spectroscopic Data of Compound 5 


\subsection{Boc-D-allo-Ile-Thz-OAllyl (24)}
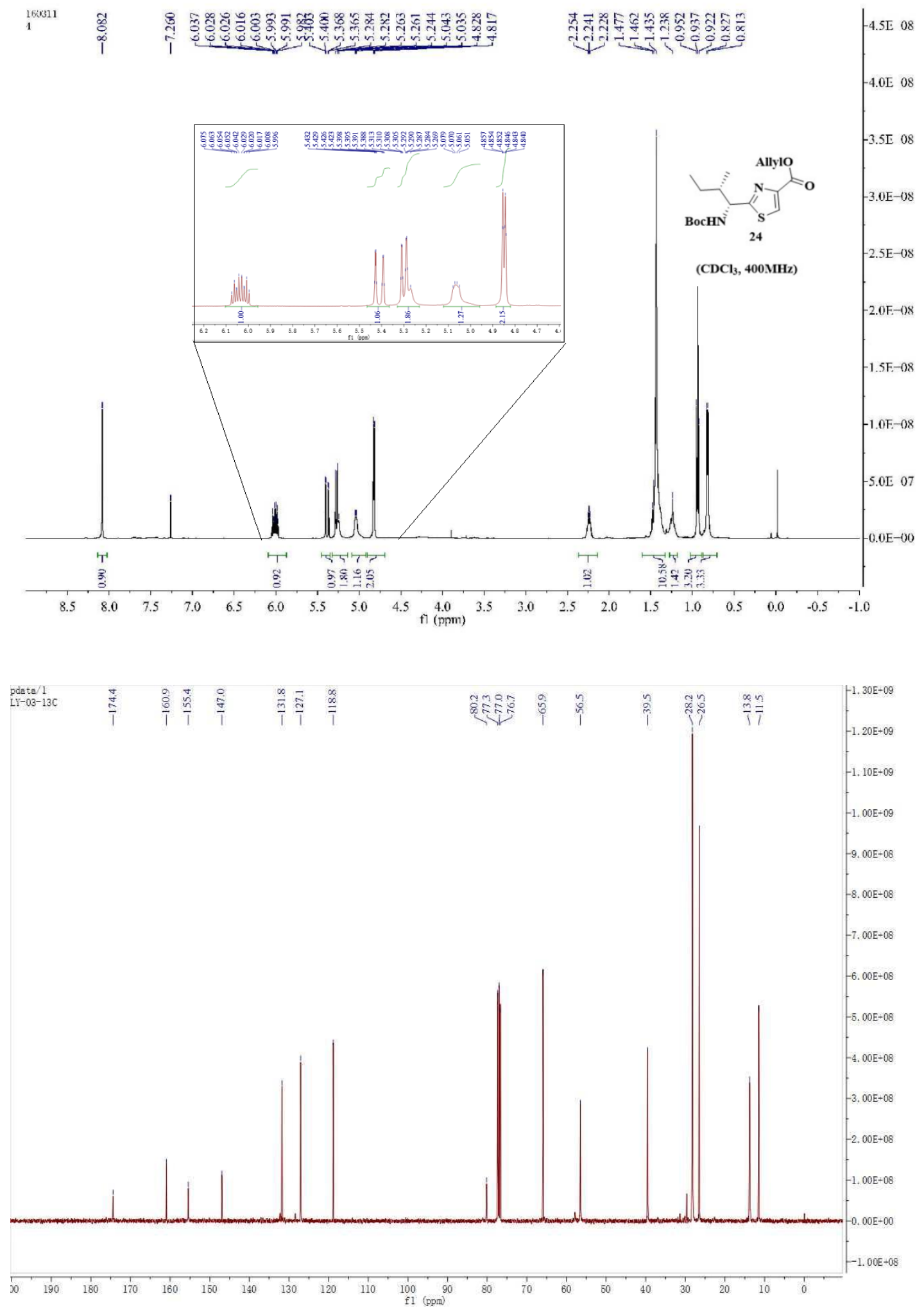

Figure S7: ${ }^{1} \mathrm{H}$ NMR and ${ }^{13} \mathrm{C}$ NMR Spectra of Compound 24 


\subsection{Thiazole $5+$ Thiazole 24}

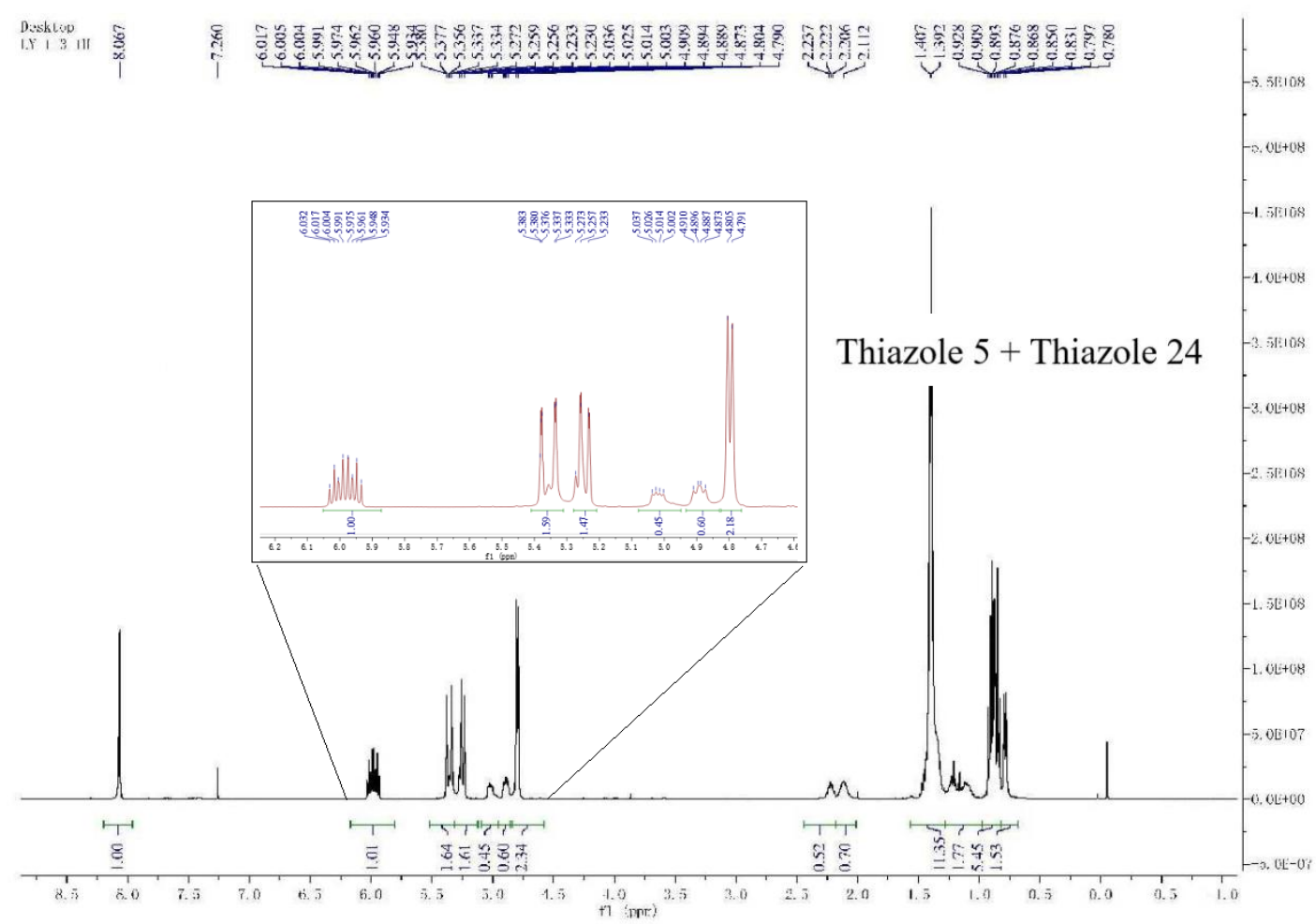

Figure S8: Comparison of ${ }^{1} \mathrm{H}$ NMR Spectroscopic Data of Compound $\mathbf{5}$ and $\mathbf{2 4}$

\subsection{Control Experiment for 25 and 26}

\section{Boc-L-Ile-Thz-OMe (25)}

Compound 5 ( $35 \mathrm{mg}, 0.1 \mathrm{mmol}$ ) was dissolved in $5 \mathrm{ml} \mathrm{MeOH}$, then $\mathrm{K}_{2} \mathrm{CO}_{3}(69 \mathrm{mg}, 0.5 \mathrm{mmol}$ ) was added into the solution and stirred for $3 \mathrm{~h}$. The reaction mixture was diluted with $15 \mathrm{ml} \mathrm{H}_{2} \mathrm{O}$ and extracted with EtOAc $(3 \times 10 \mathrm{ml})$. The combined organic phase was washed with saturated brine, dried $\left(\mathrm{Na}_{2} \mathrm{SO}_{4}\right)$, concentrated and the residue purified by flash chromatography to give the desired $\mathbf{2 5}$ in $100 \%$ yield.

\section{Cbz-Gly-L-Thr-L-Phe-L-Thr-OMe (26)}

Route A Compound 20 (33 mg, $0.05 \mathrm{mmol}$ ) was dissolved in $8 \mathrm{ml} \mathrm{DCM}$, then $4 \mathrm{ml}$ TFA was added into the mixture slowly at $0{ }^{\circ} \mathrm{C}$. Then the mixture was warmed to room temperature and stirred until TLC showed complete consumption of the starting material $(\sim 20 \mathrm{~h})$. The organic phase was concentrated under reduced pressure and the residue was purified by flash column chromatography $\left(\mathrm{CH}_{2} \mathrm{Cl}_{2} / \mathrm{MeOH}, 15: 1\right)$ to give 26 as a colorless oil ( $25 \mathrm{mg}, 86 \%)$.

Route B Following the general method B, compound $\mathbf{2 6}$ was obtained in $78 \%$ yield from dipeptide $\mathbf{1 6}$ and 18. 

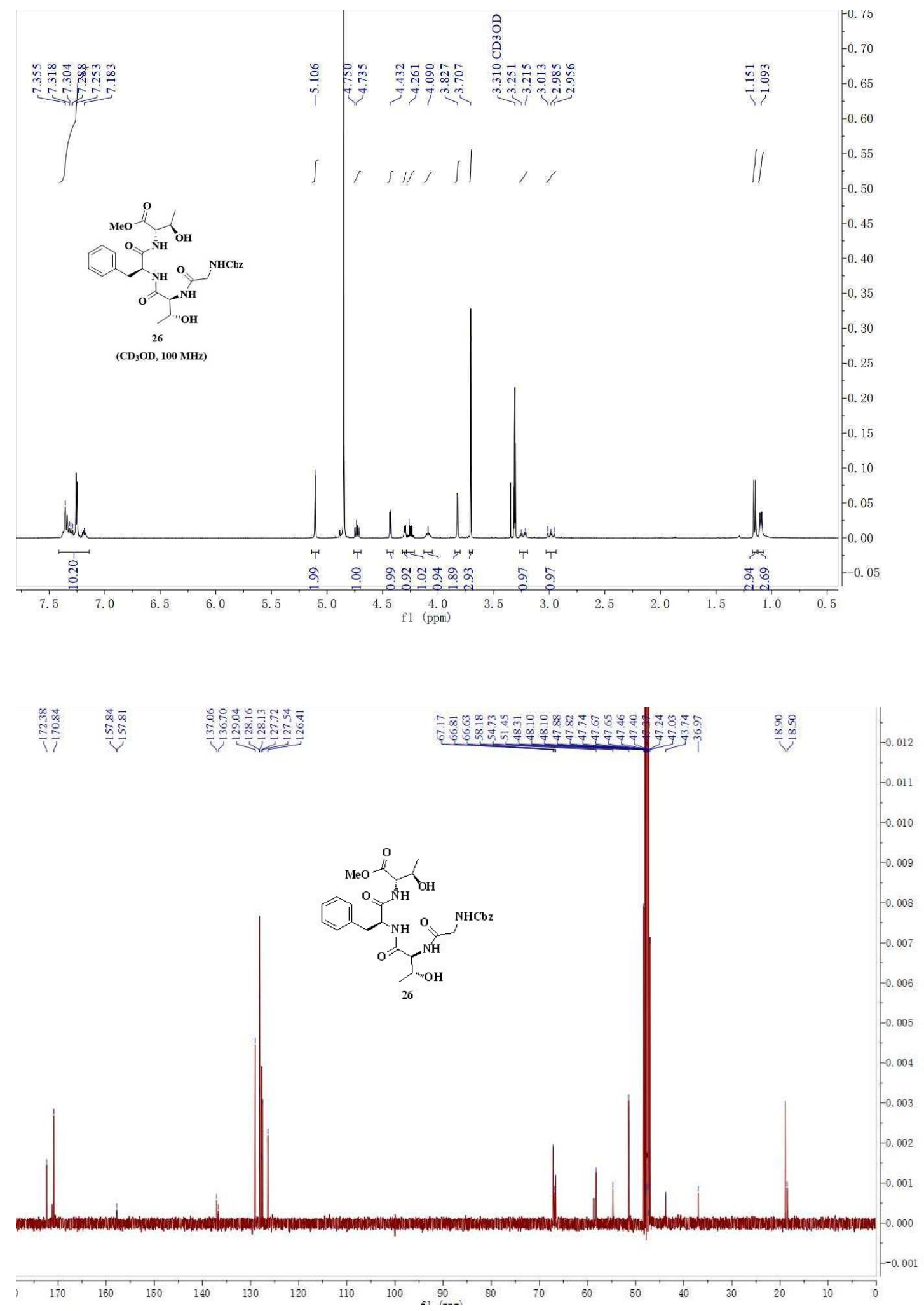

Figure S9: Copy of ${ }^{1} \mathrm{H}$ NMR and ${ }^{13} \mathrm{C}$ NMR Spectra of Compound 26 
5. Copies of NMR Spectra of Synthesized Compounds.

Cbz-Gly-L-Thr-OMe (16)

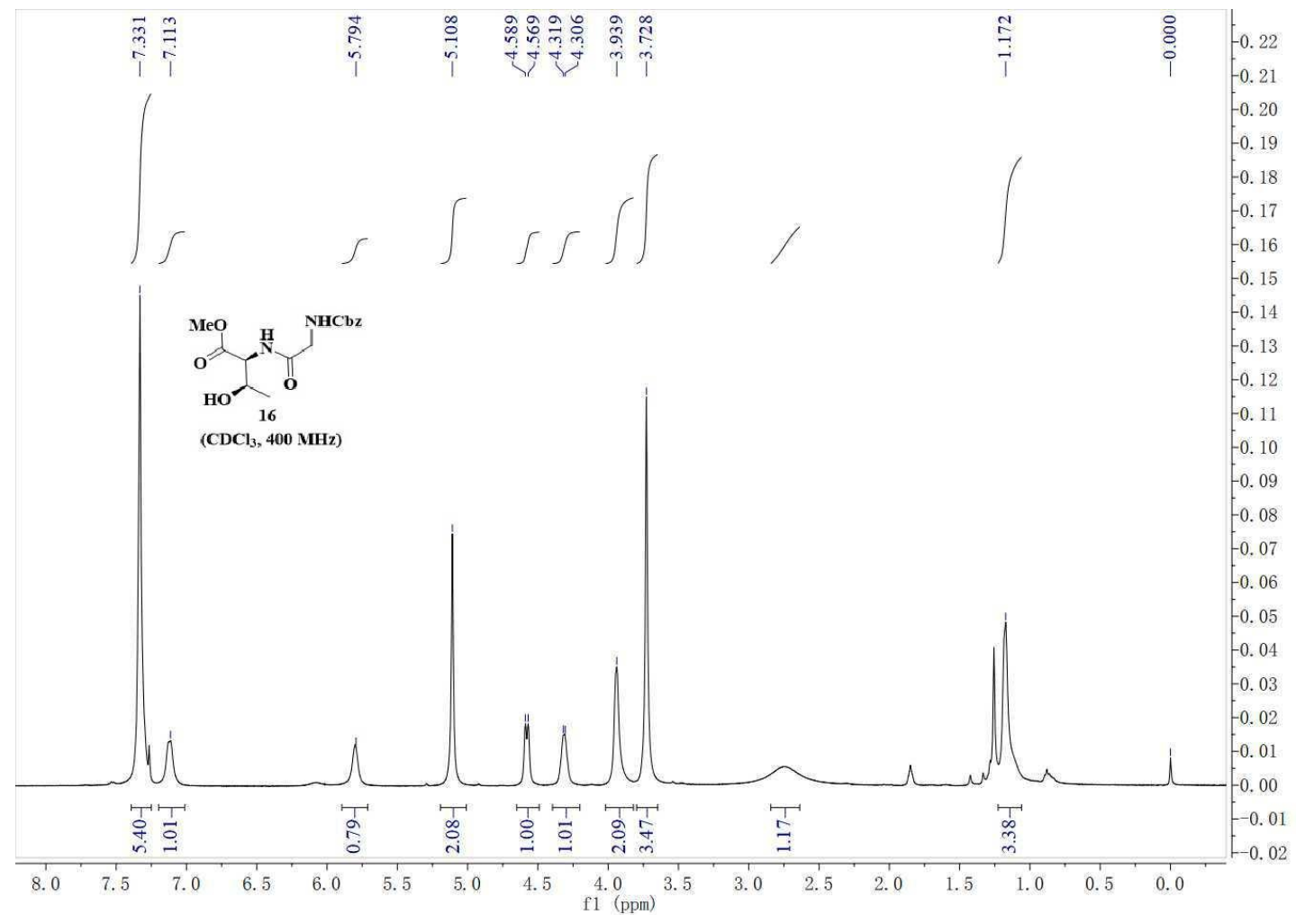

Cbz-Gly-L-Thr( $\psi$ Me,MePro)-OMe (17)

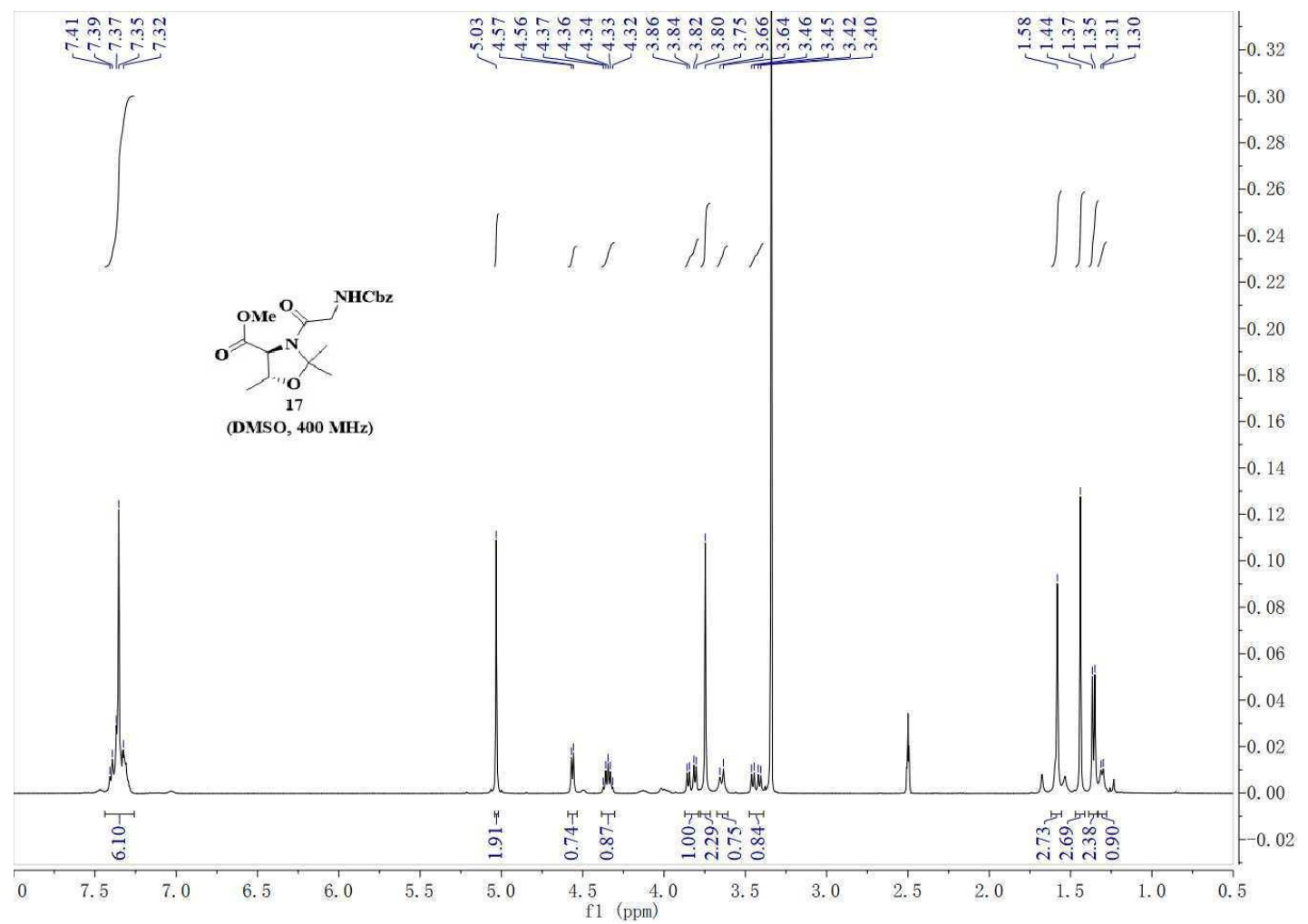


Boc-L-Phe-L-Thr-OMe (18)
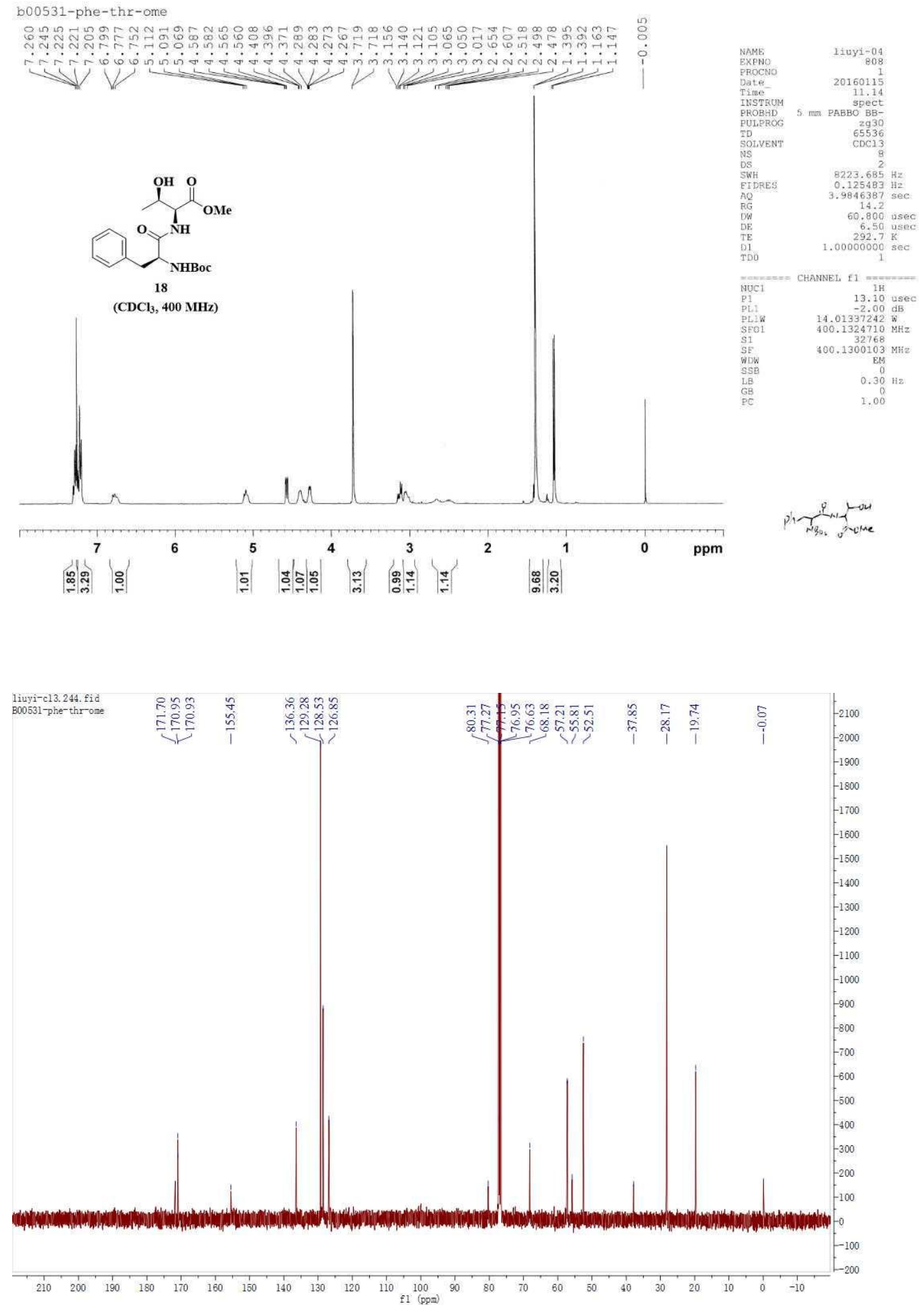
Cbz-Gly-L-Thr( $\psi^{\mathrm{Me}, \mathrm{Me}}$ Pro)-L-Phe-L-Thr-OMe (19)

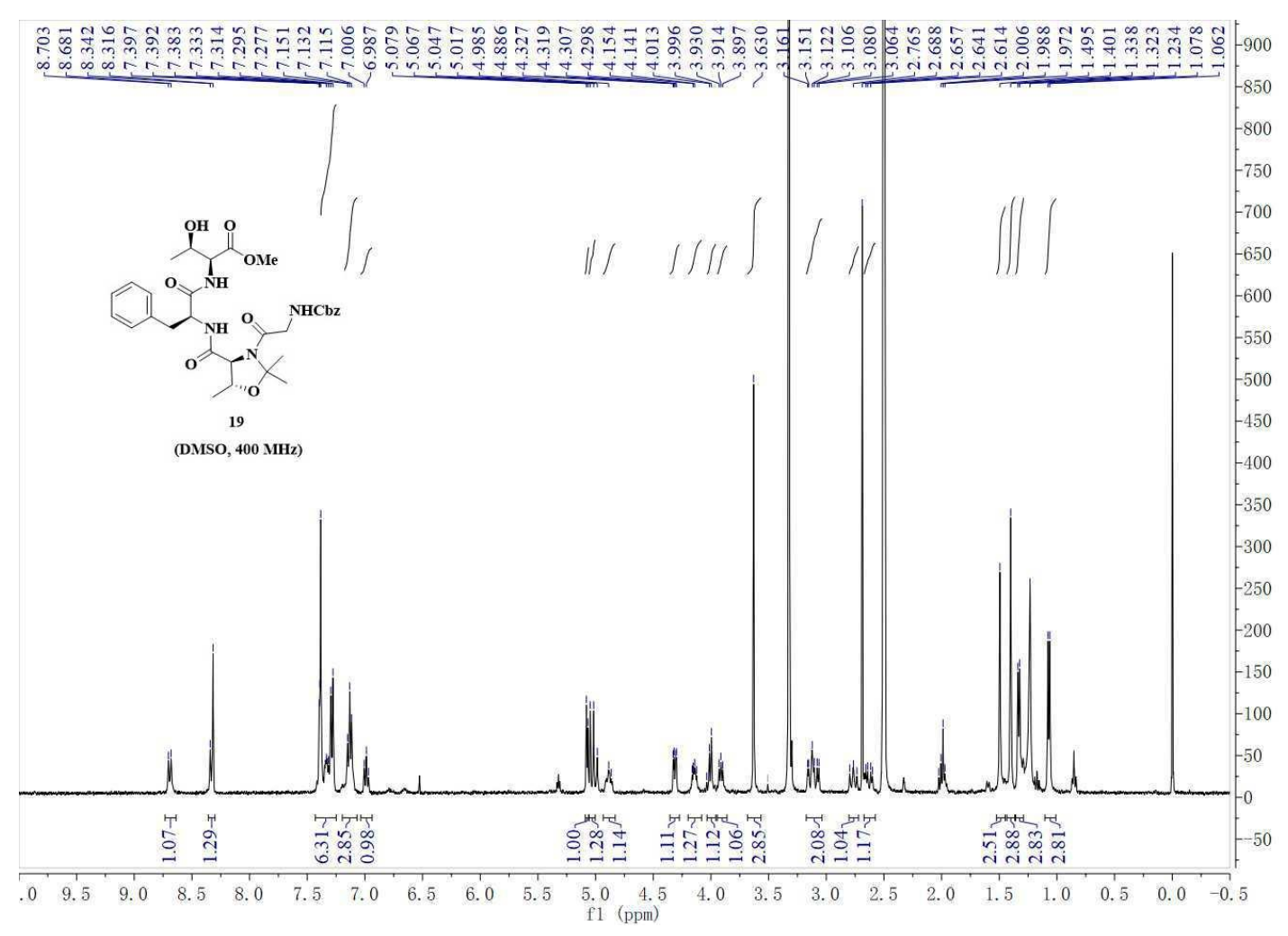

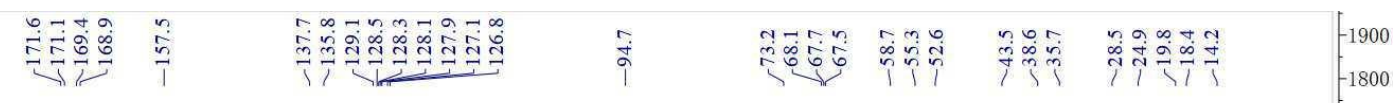

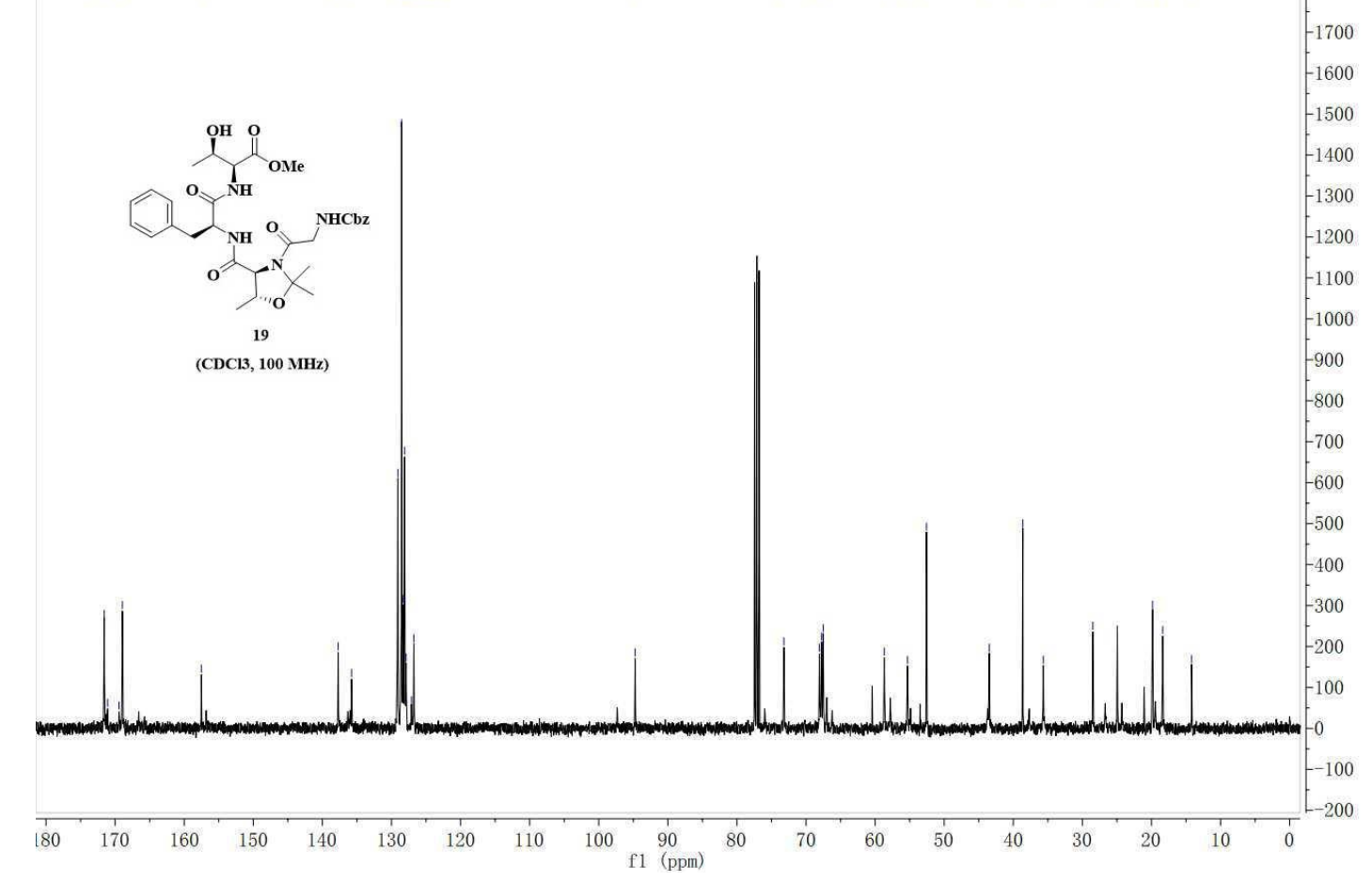



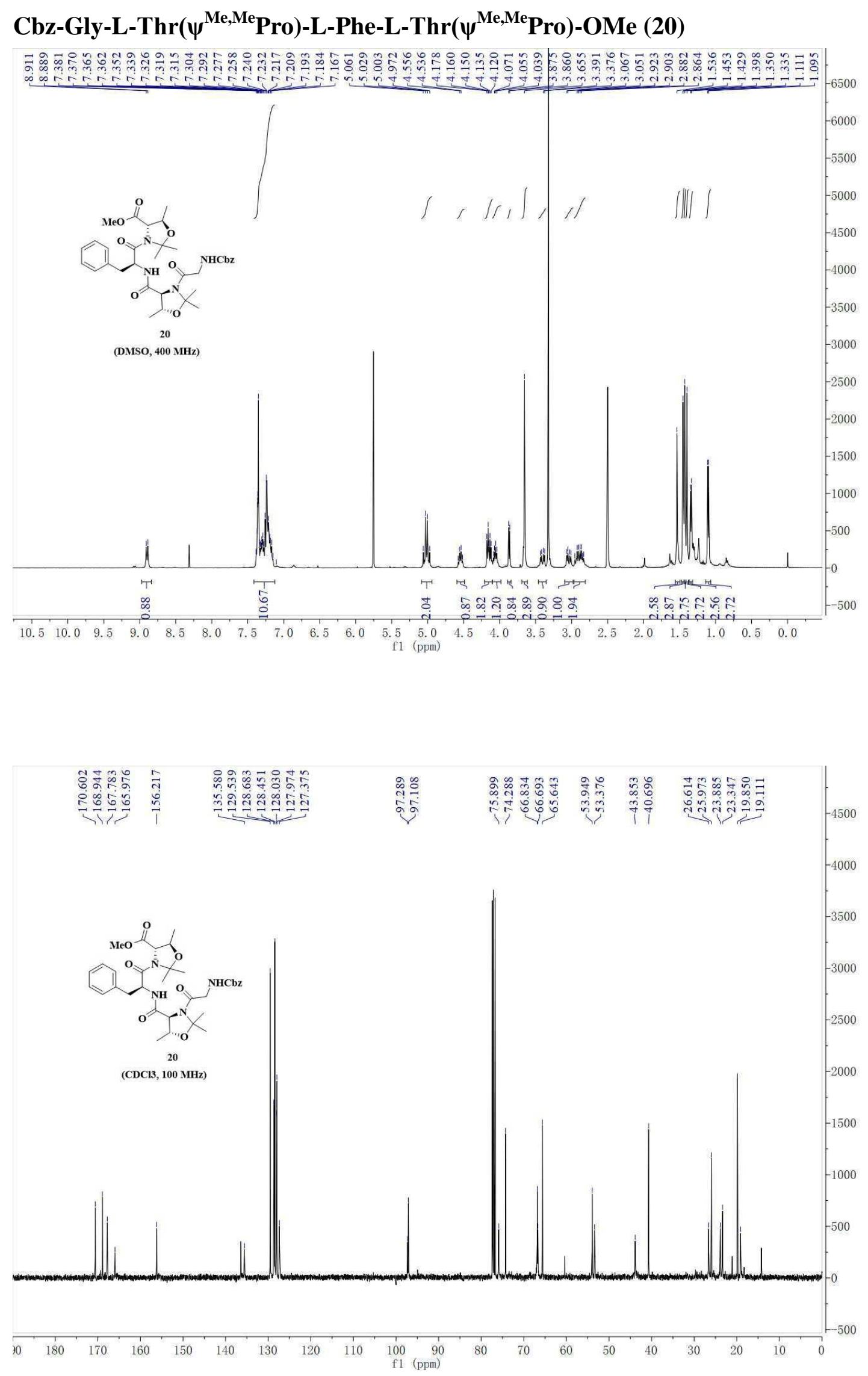


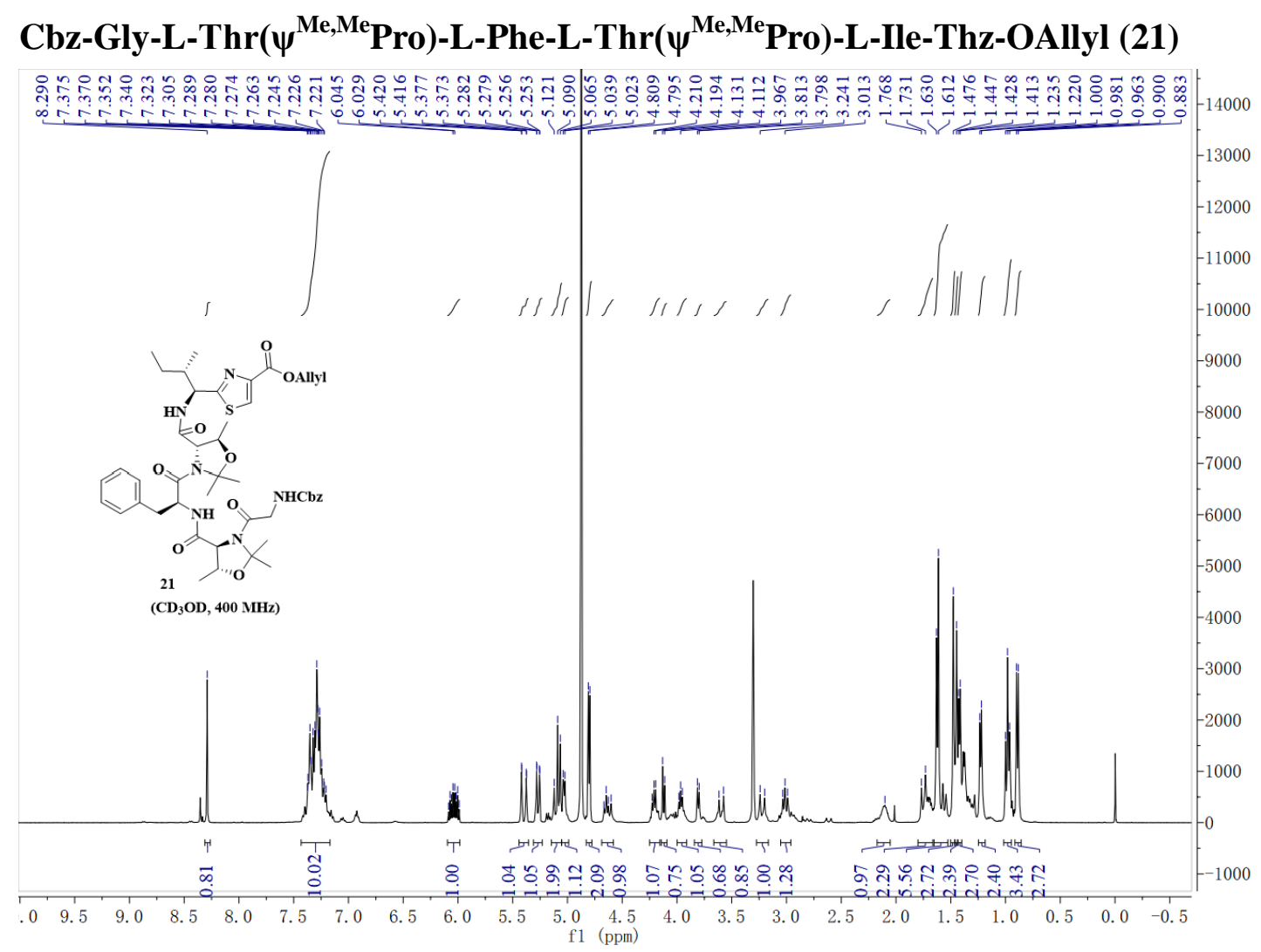

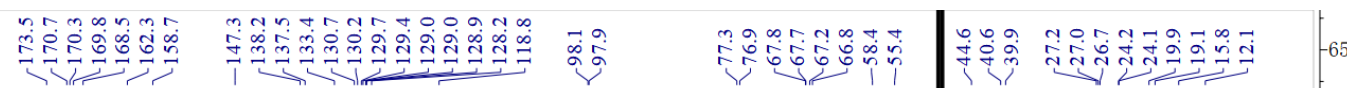

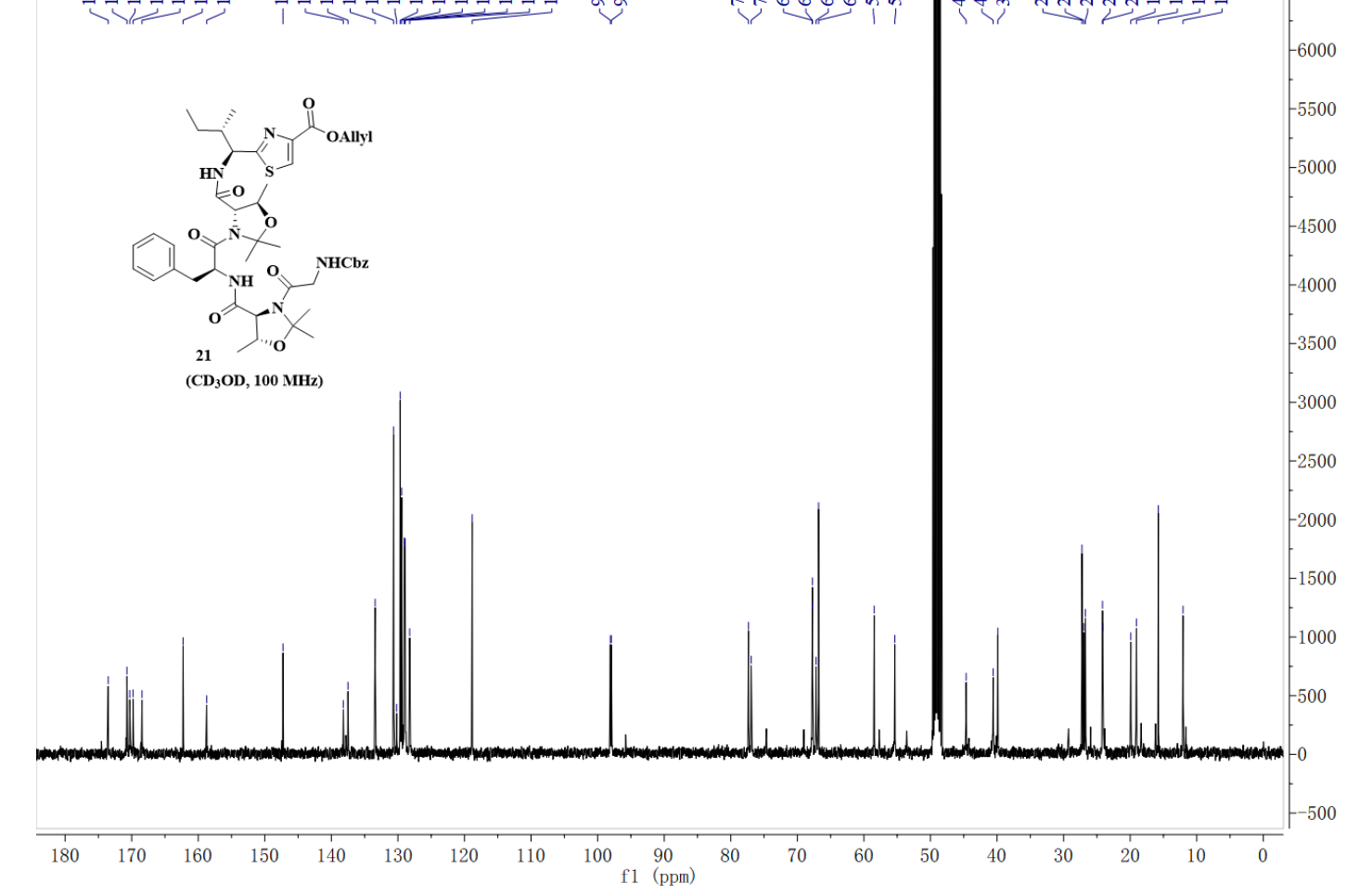



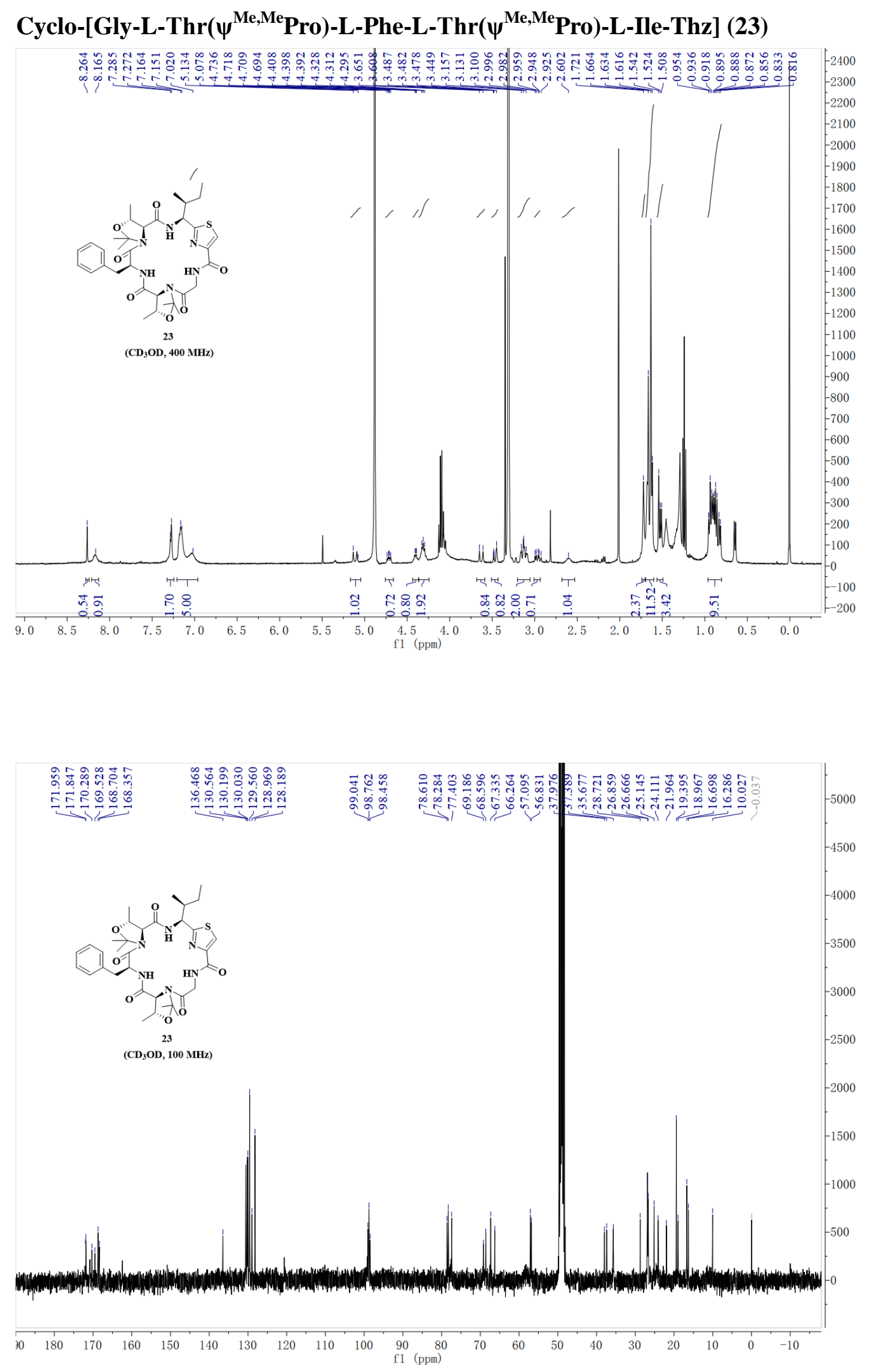


\section{cyclo-[L-Thr-L-Phe-L-Thr-Gly-L-Ile(Thz)] (1)}
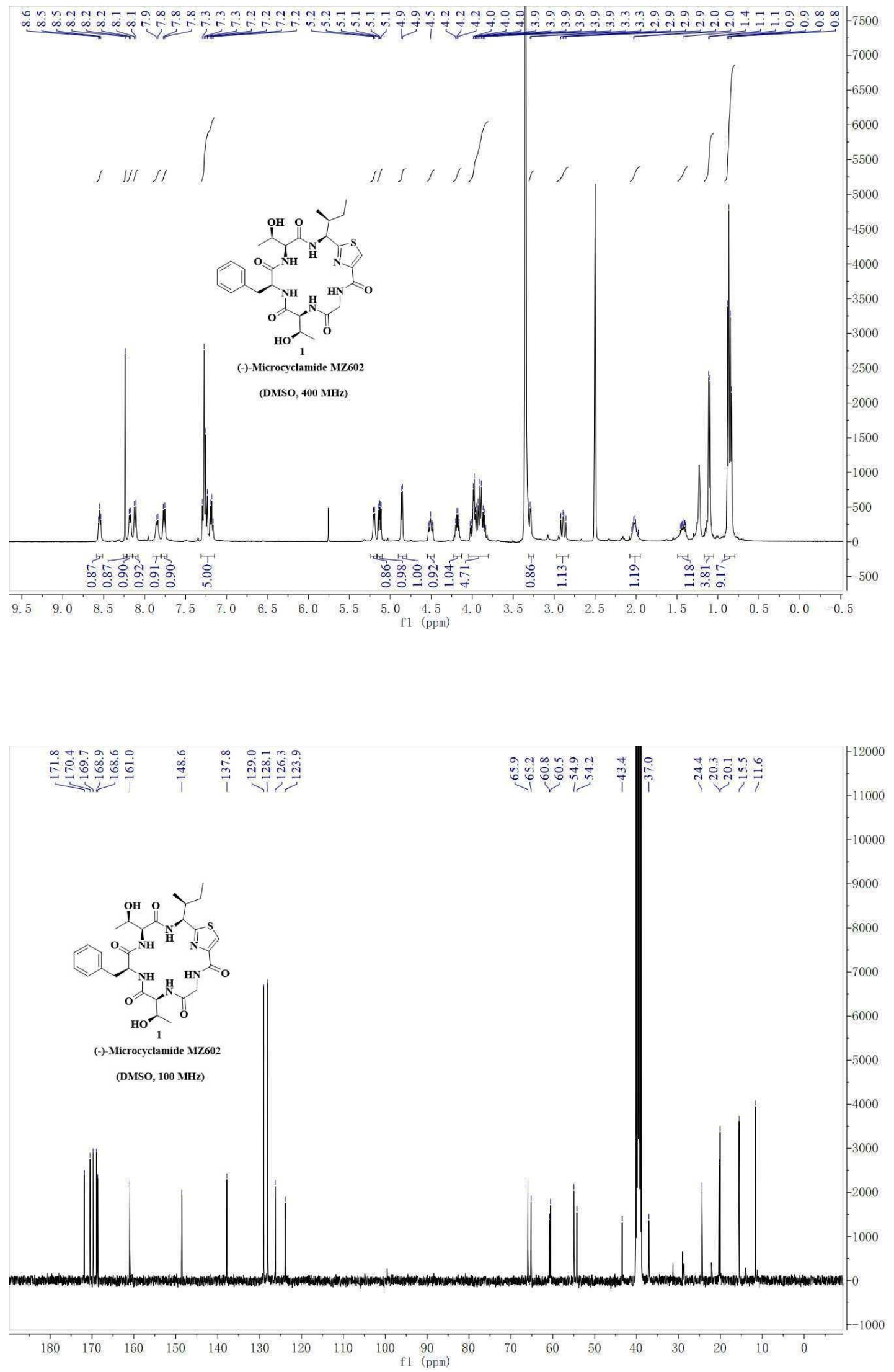


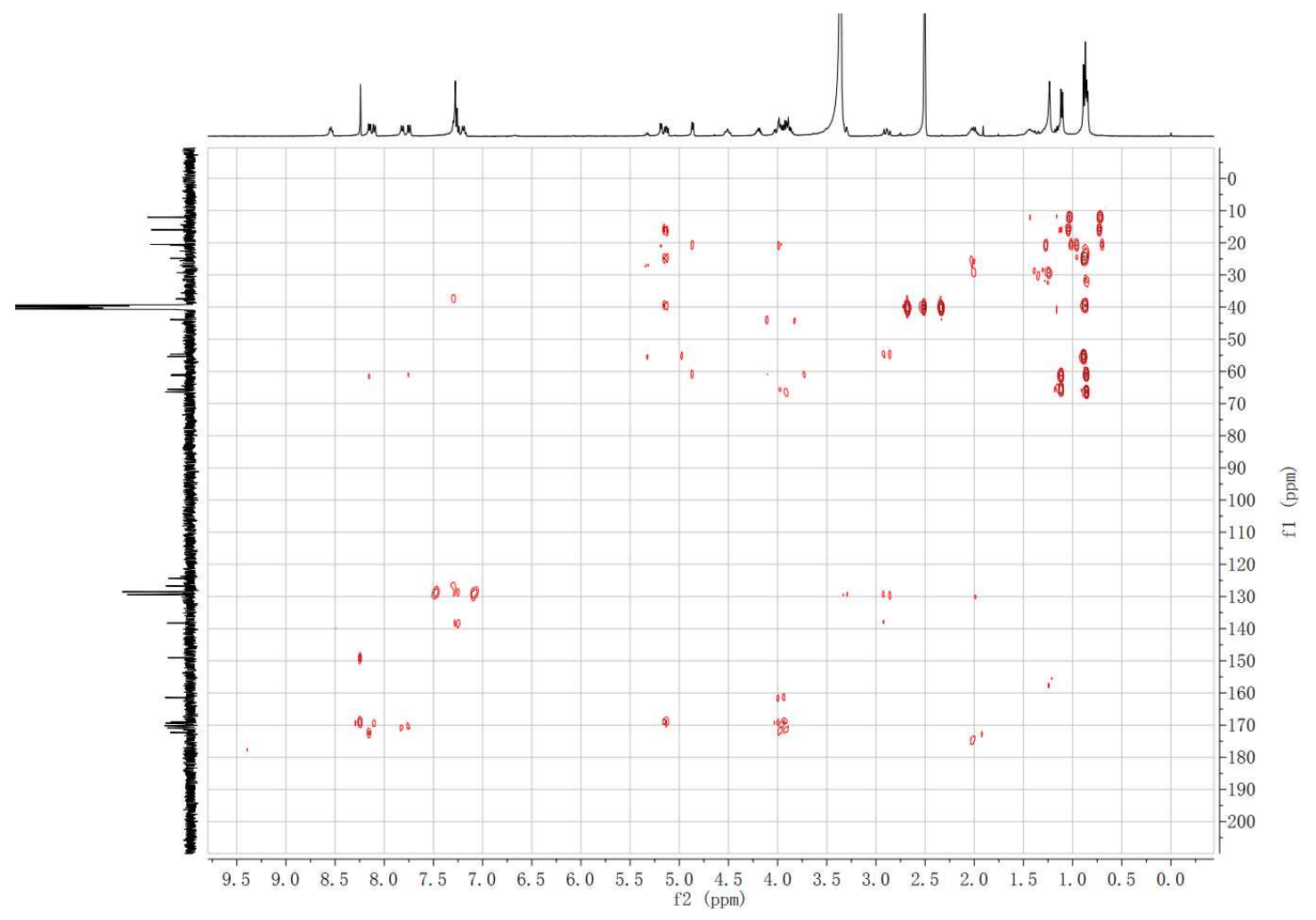

S23 
Boc-L-Phe-L-Thr(Bzl)-OAllyl (3)
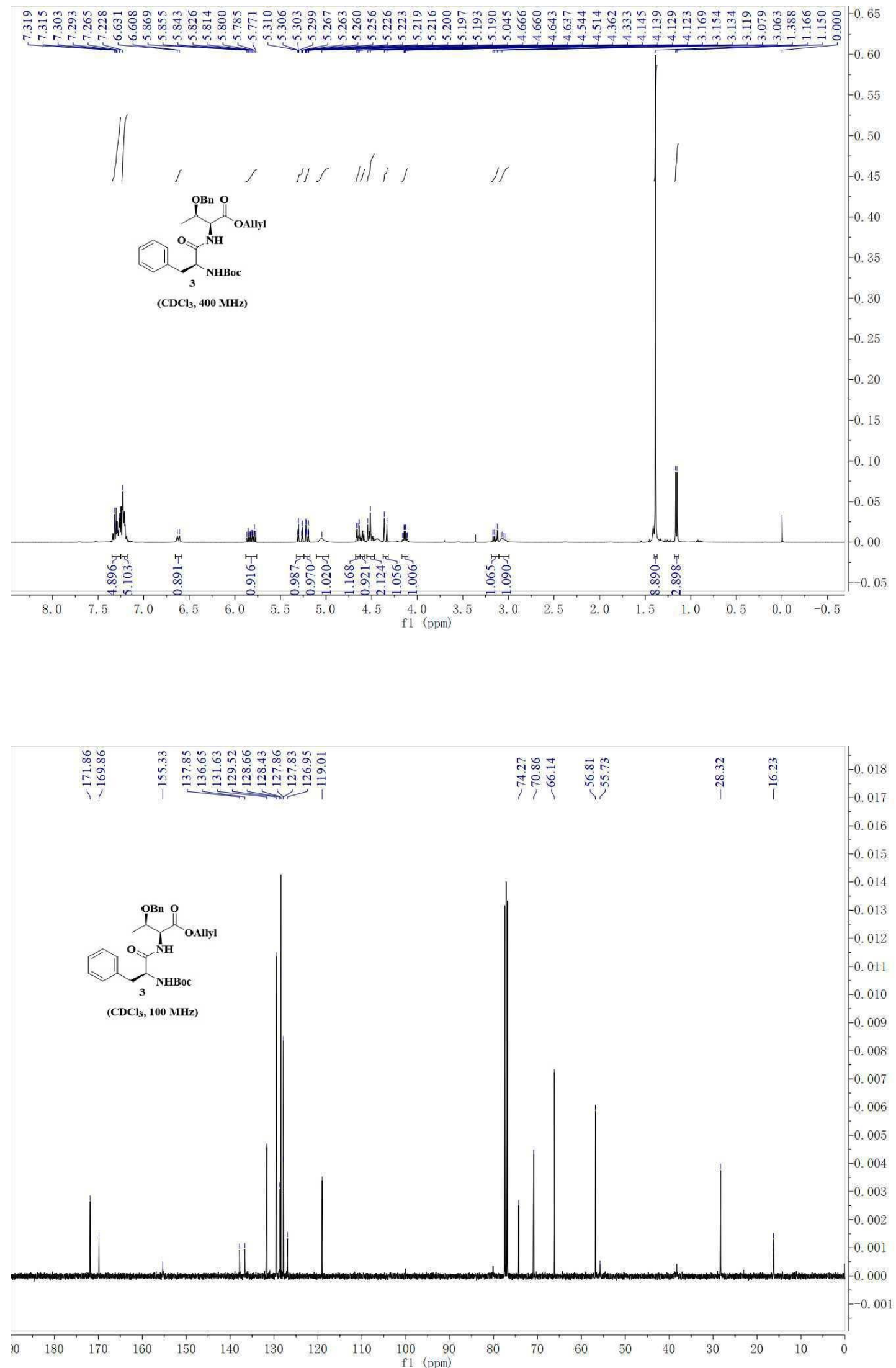
Boc-Gly-L-Thr(Bzl)-OAllyl (4)
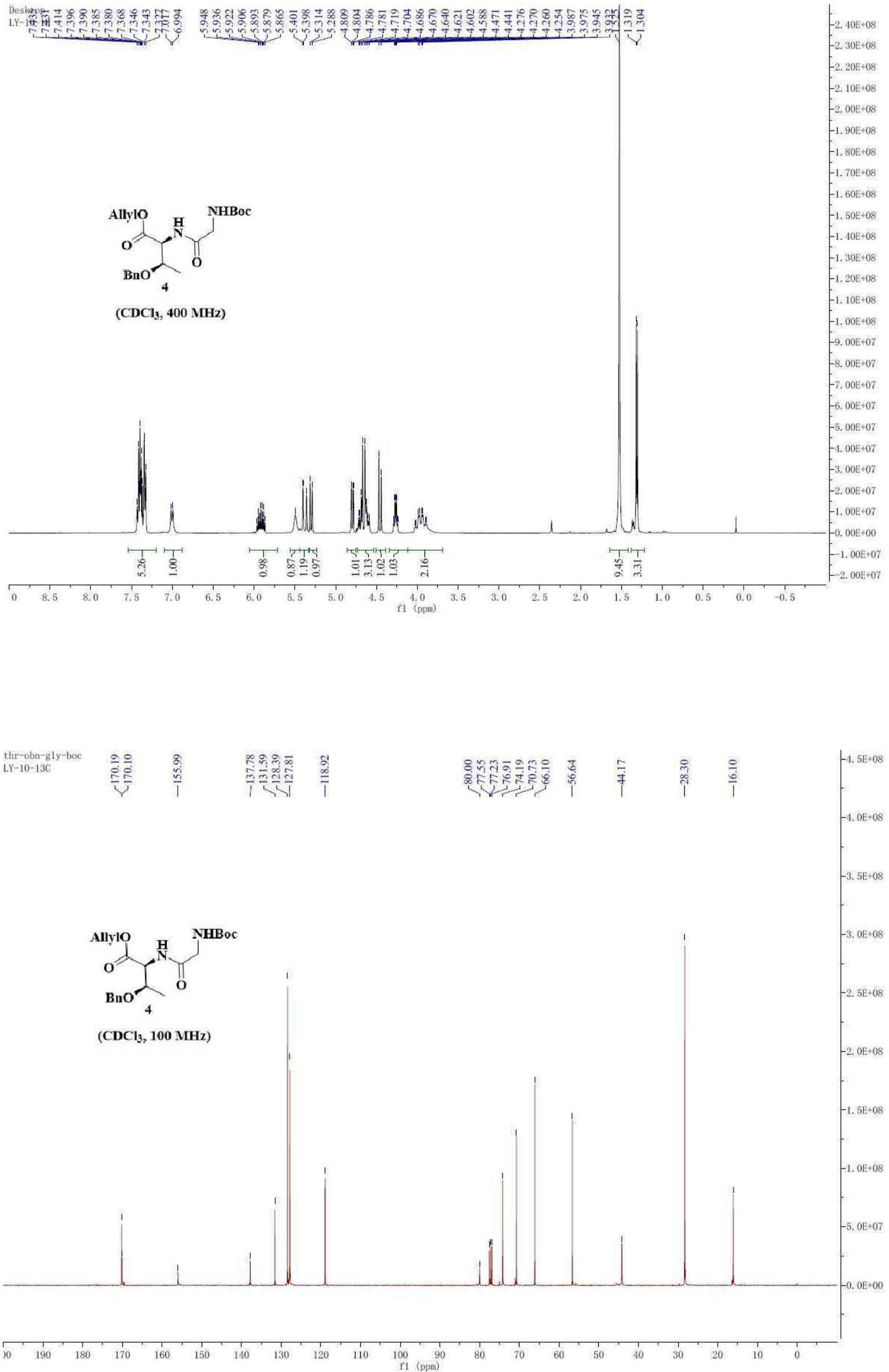


\section{Boc-Gly-L-Thr(Bzl)-L-Phe-L-Thr(Bzl)-OAllyl (11)}
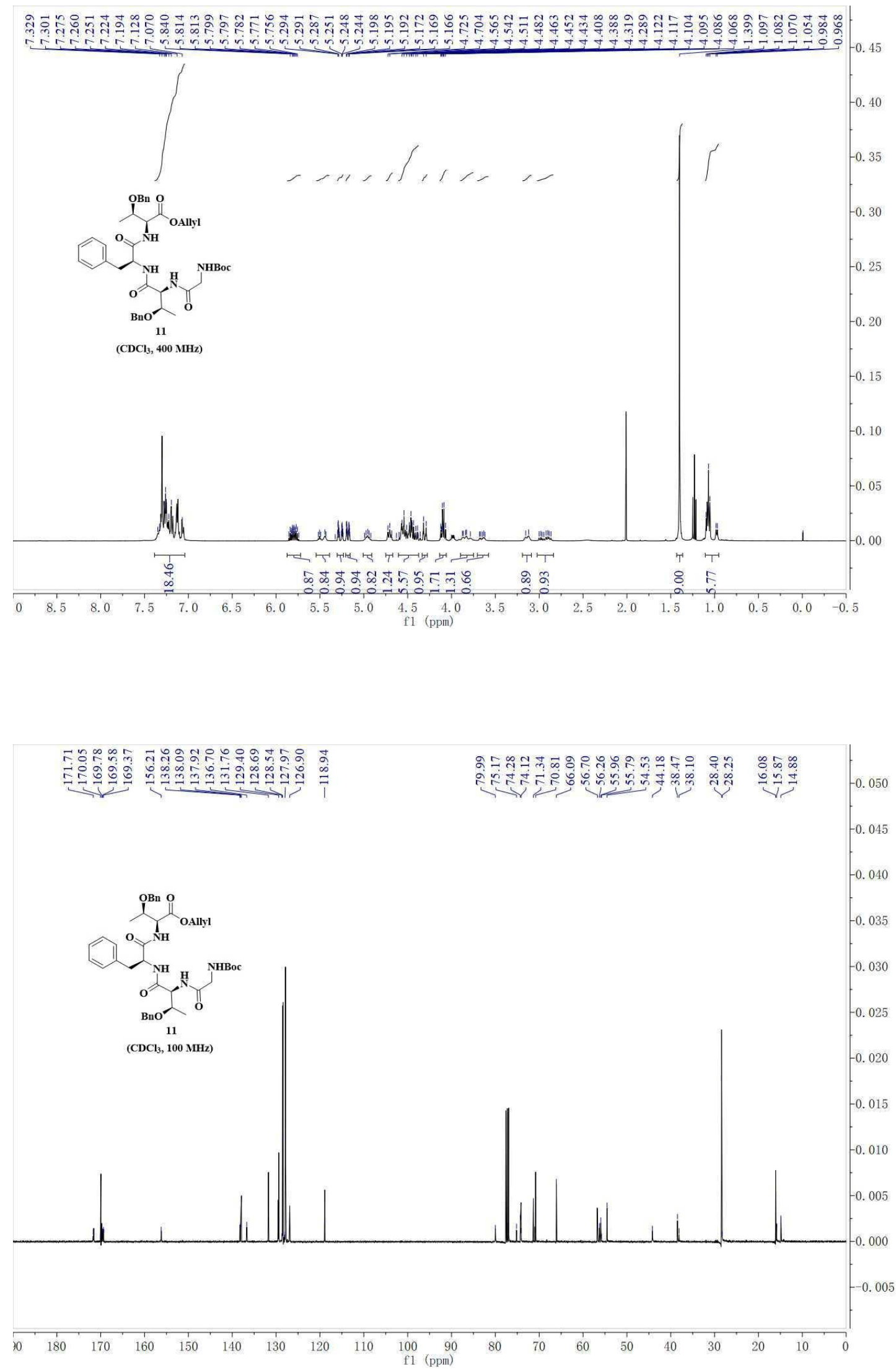
Boc-Gly-L-Thr(Bzl)-L-Phe-L-Thr(Bzl)-L-Ile-Thz-OAllyl (12)
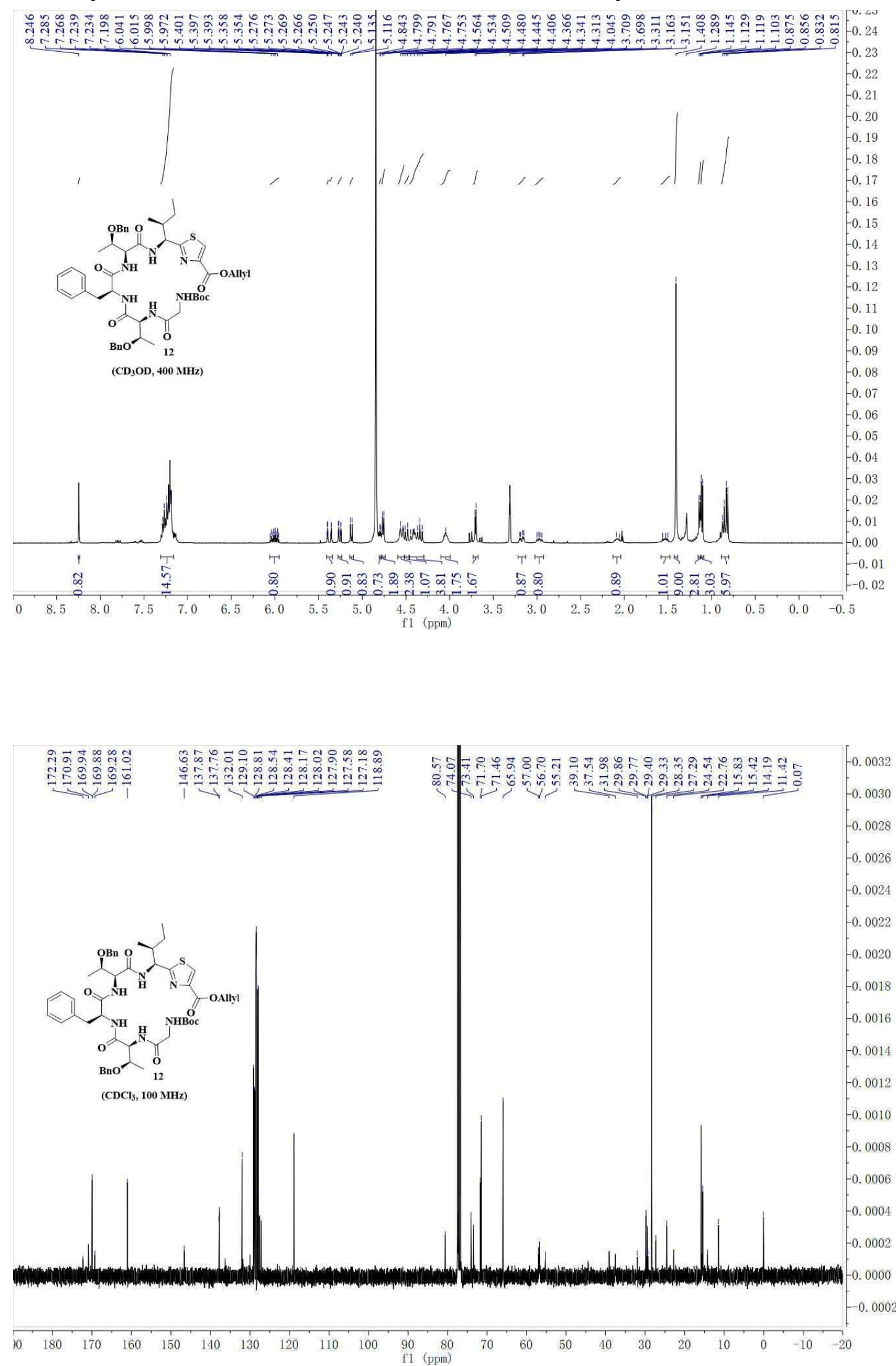
Cbz-L-Ala-L-Thr-OMe (28)

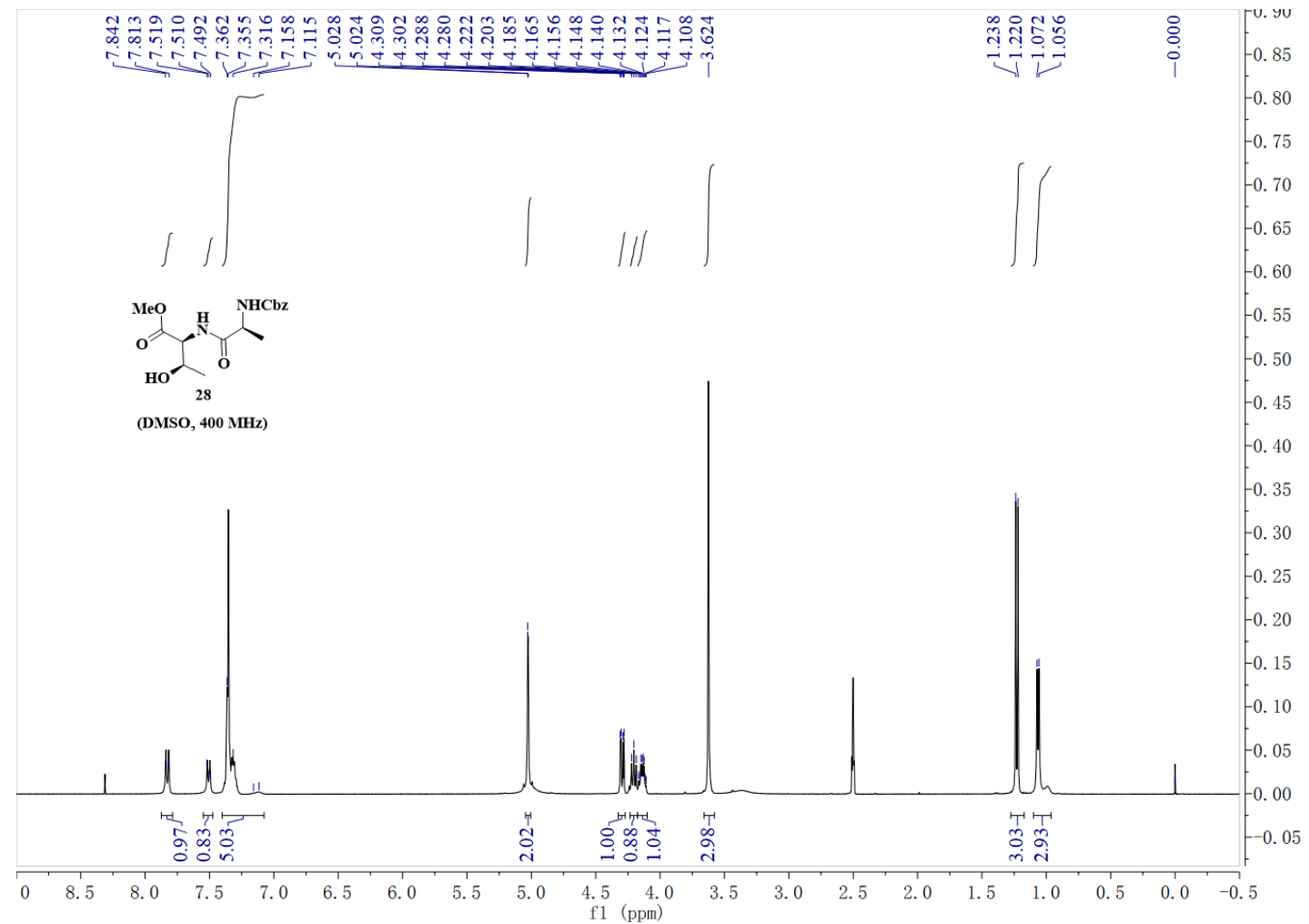

\section{Cbz-L-Ala-L-Thr( $\psi^{\mathrm{Me}, \mathrm{Me}}$ Pro)-OMe (29)}

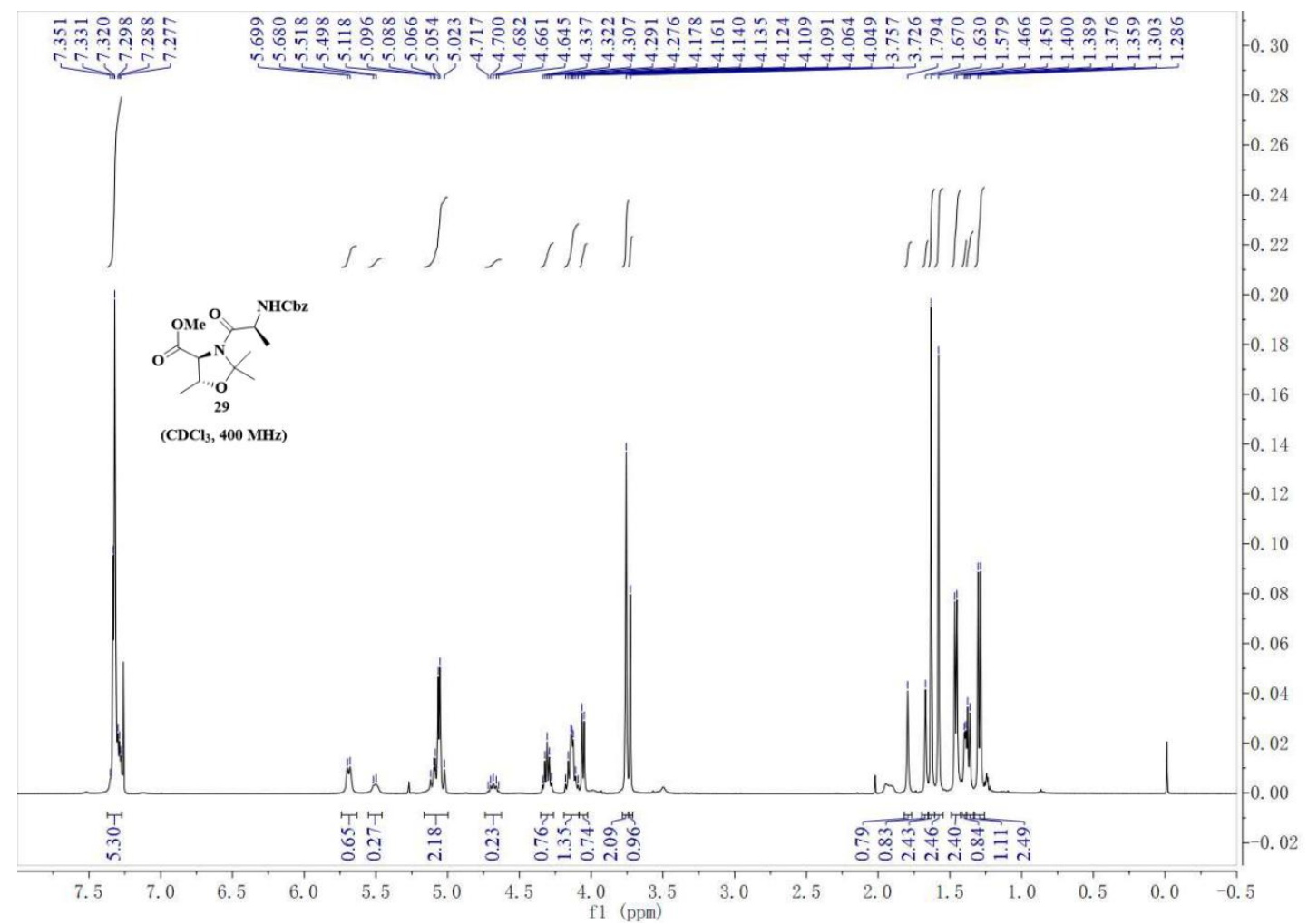




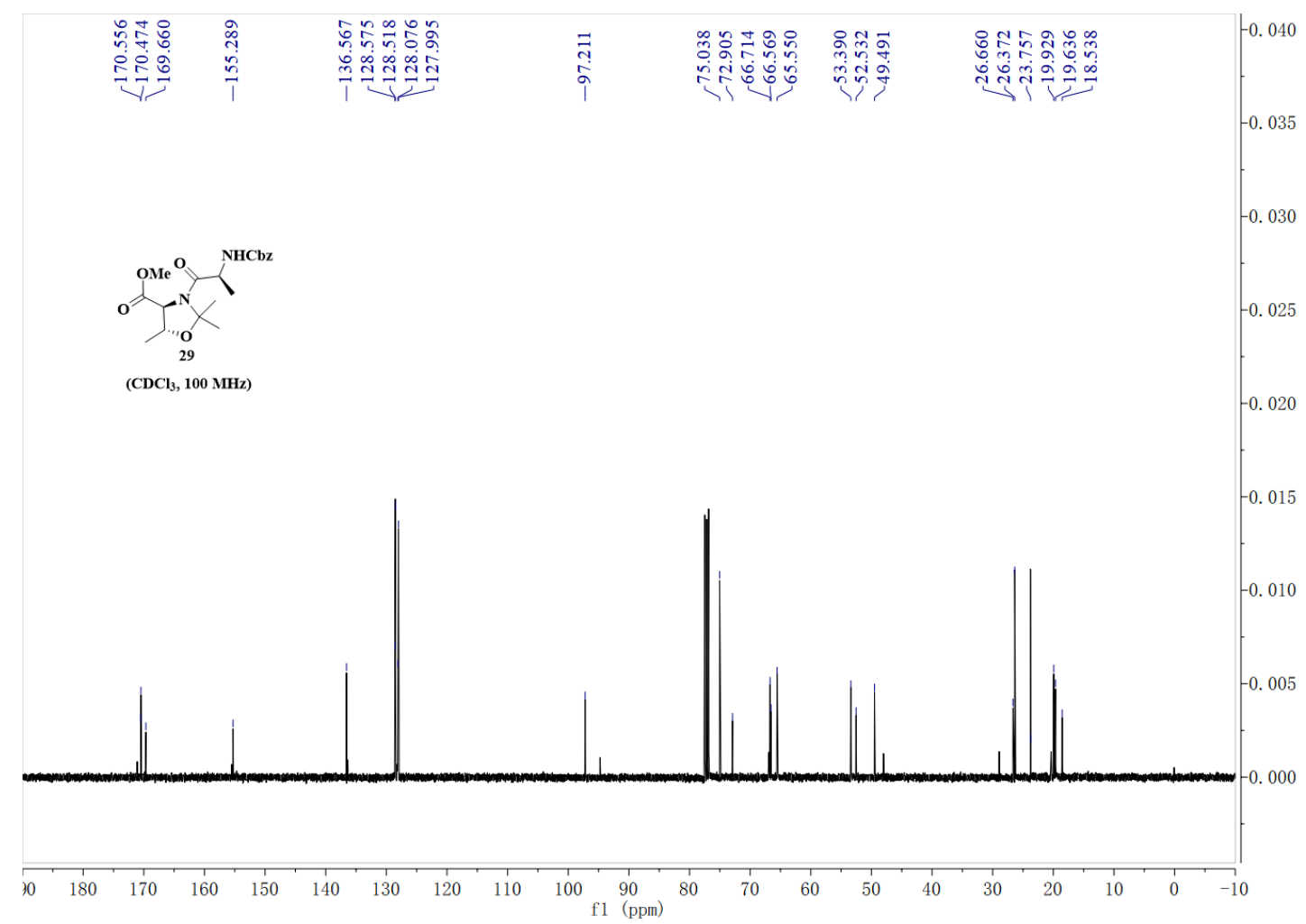

Boc-L-Val-L-Thr-OMe (31)

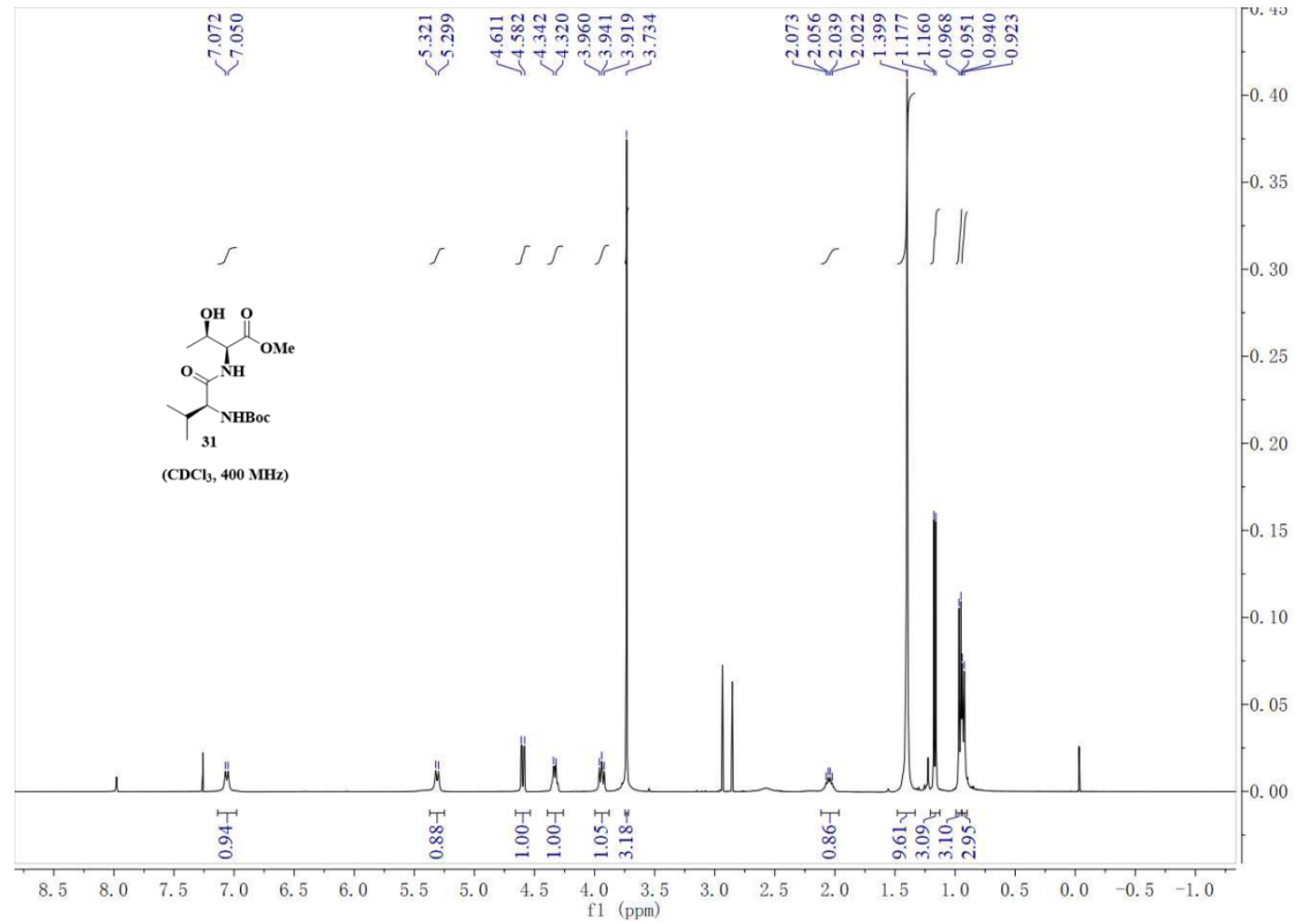




\section{Cbz-L-Ala-L-Thr( $\psi^{\mathrm{Me}, \mathrm{Me}}$ Pro)-L-Val-L-Thr-OMe (32)}
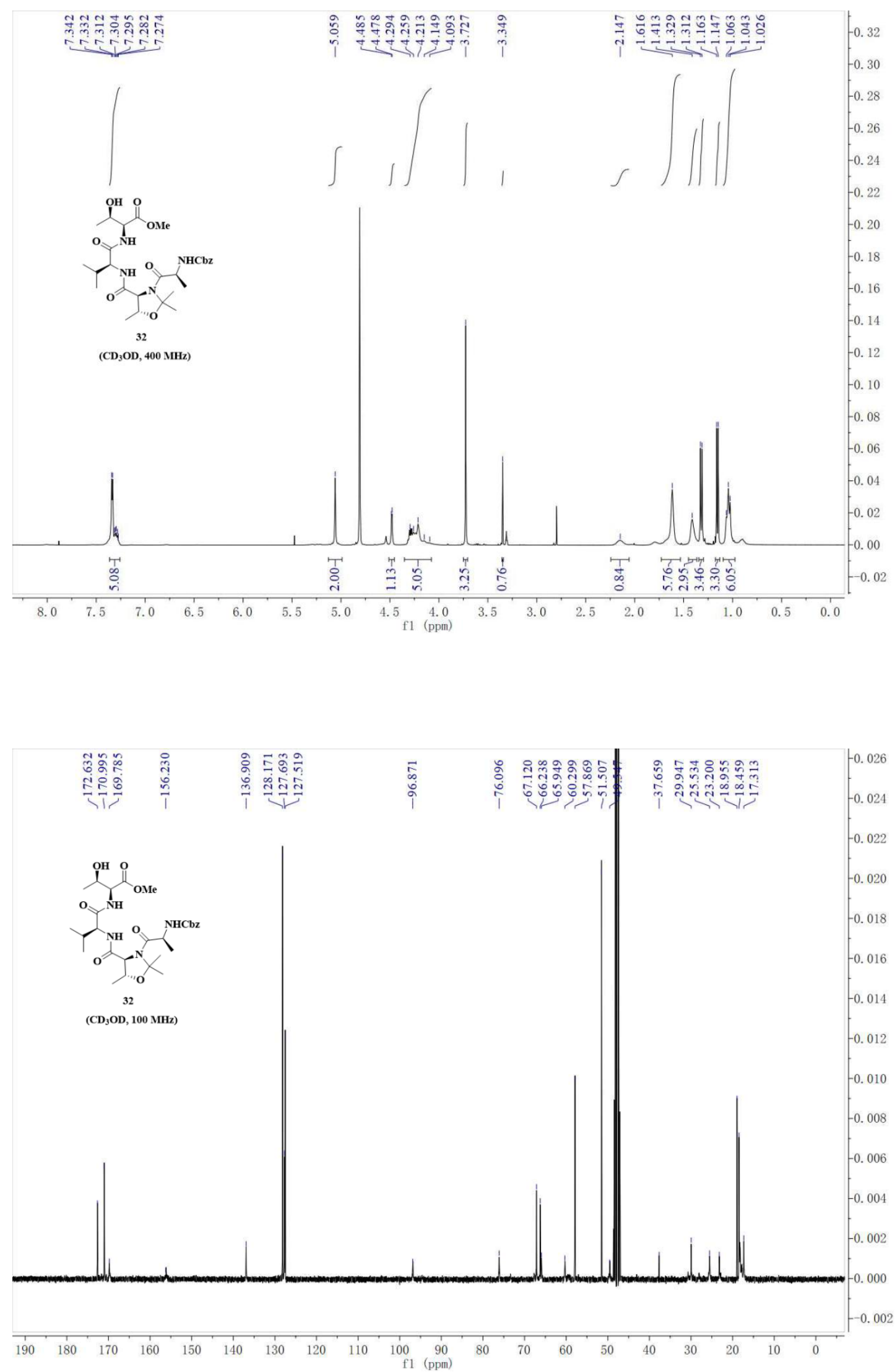
Cbz-L-Ala-L-Thr( $\psi^{\mathrm{Me}, \mathrm{Me}}$ Pro)-L-Val-L-Thr( $\psi^{\mathrm{Me}, \mathrm{Me}}$ Pro)-OMe (33)
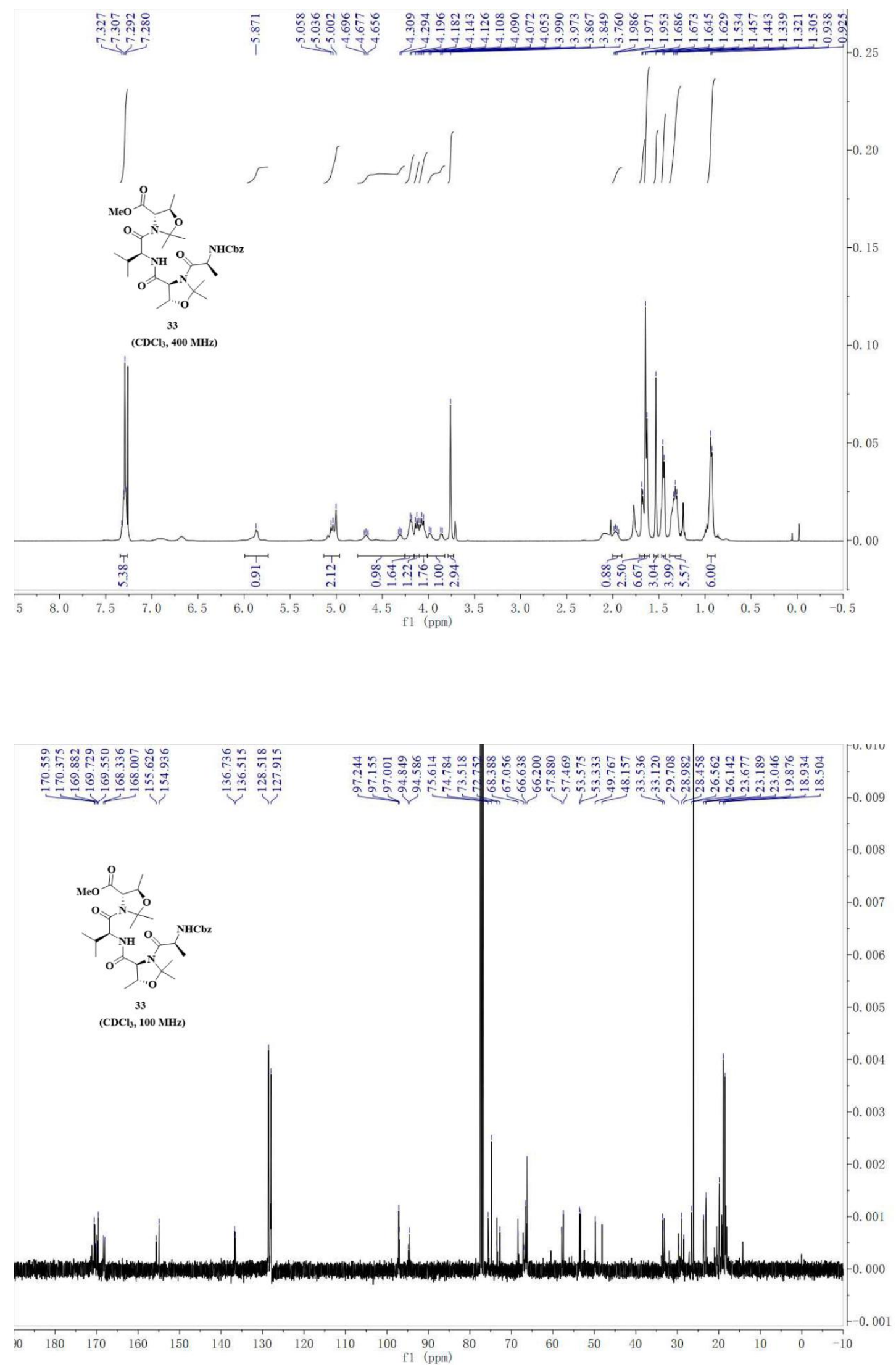
Cbz-L-Ala-L-Thr( $\psi^{\mathrm{Me}, \mathrm{Me}}$ Pro)-L-Val-L-Thr( $\psi^{\mathrm{Me}, \mathrm{Me}}$ Pro)-L-Ile-Thz-OAllyl (34)
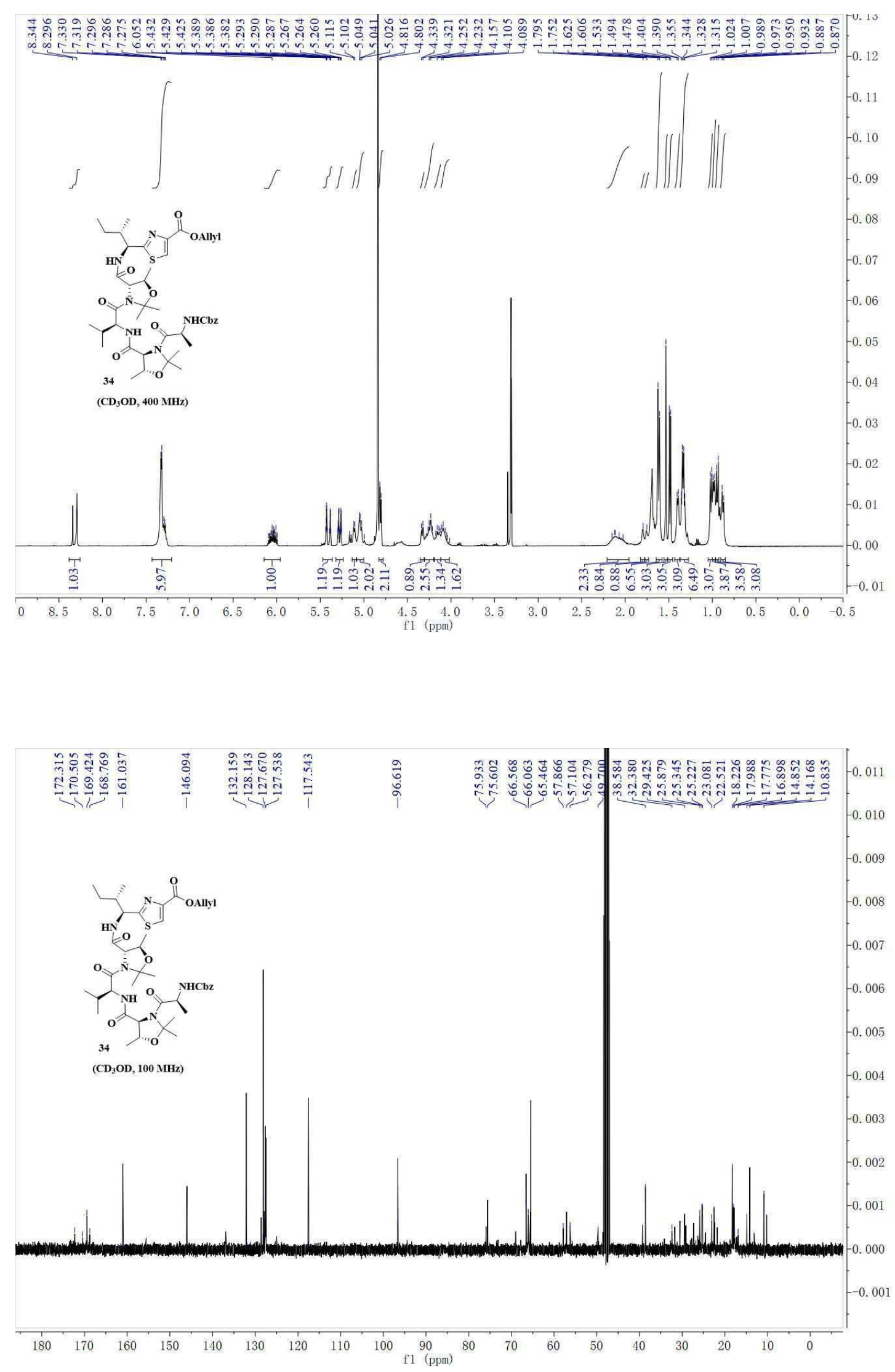
Cyclo-[L-Ala-L-Thr( $\psi^{\text {Me,Me }}$ Pro)-L-Val-L-Thr( $\psi^{\text {Me,Me }}$ Pro)-L-Ile-Thz] (36)
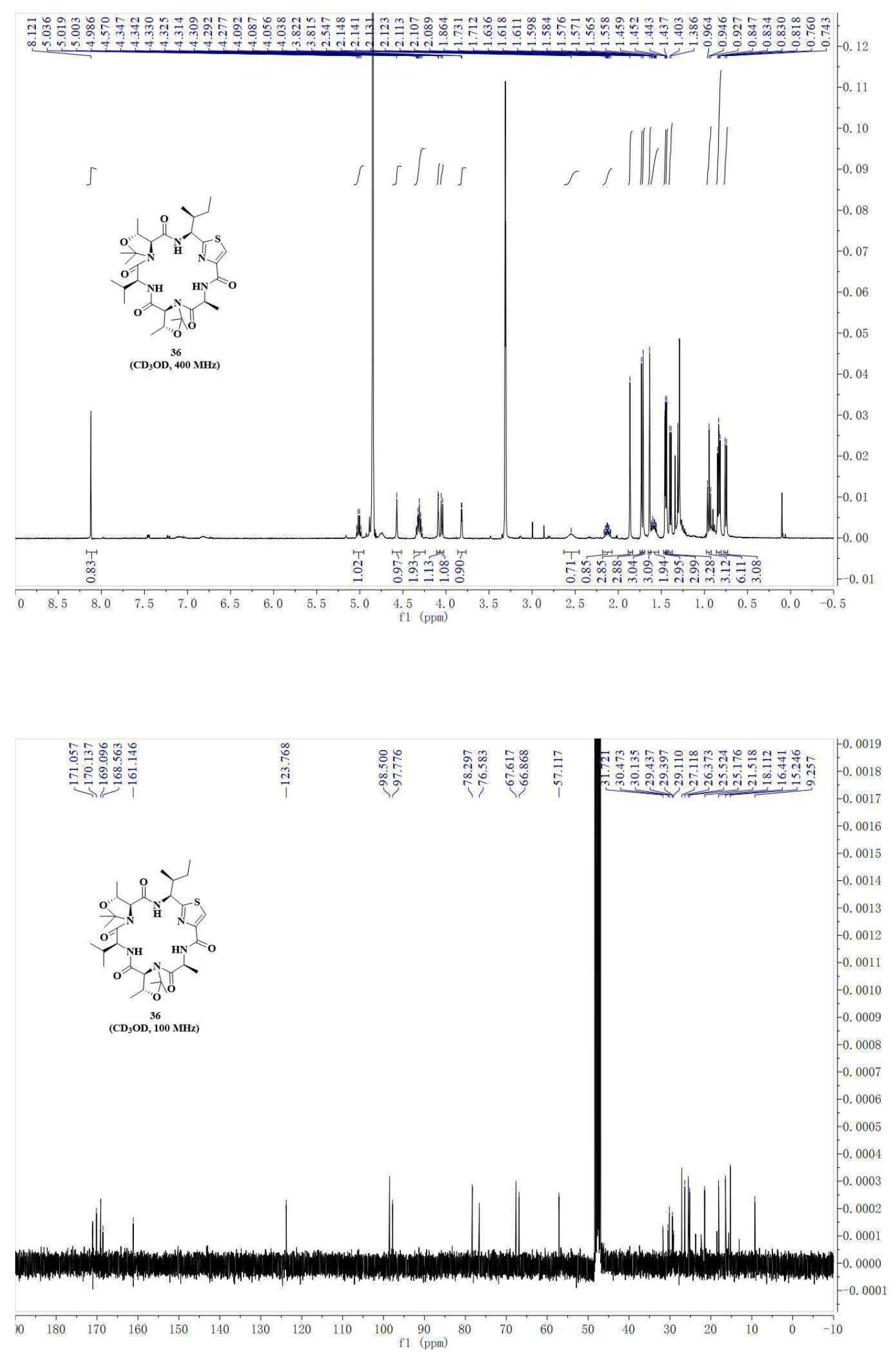
cyclo-[L-Thr-L-Val-L-Thr-L-Ala-L-Ile(Thz)] (2)
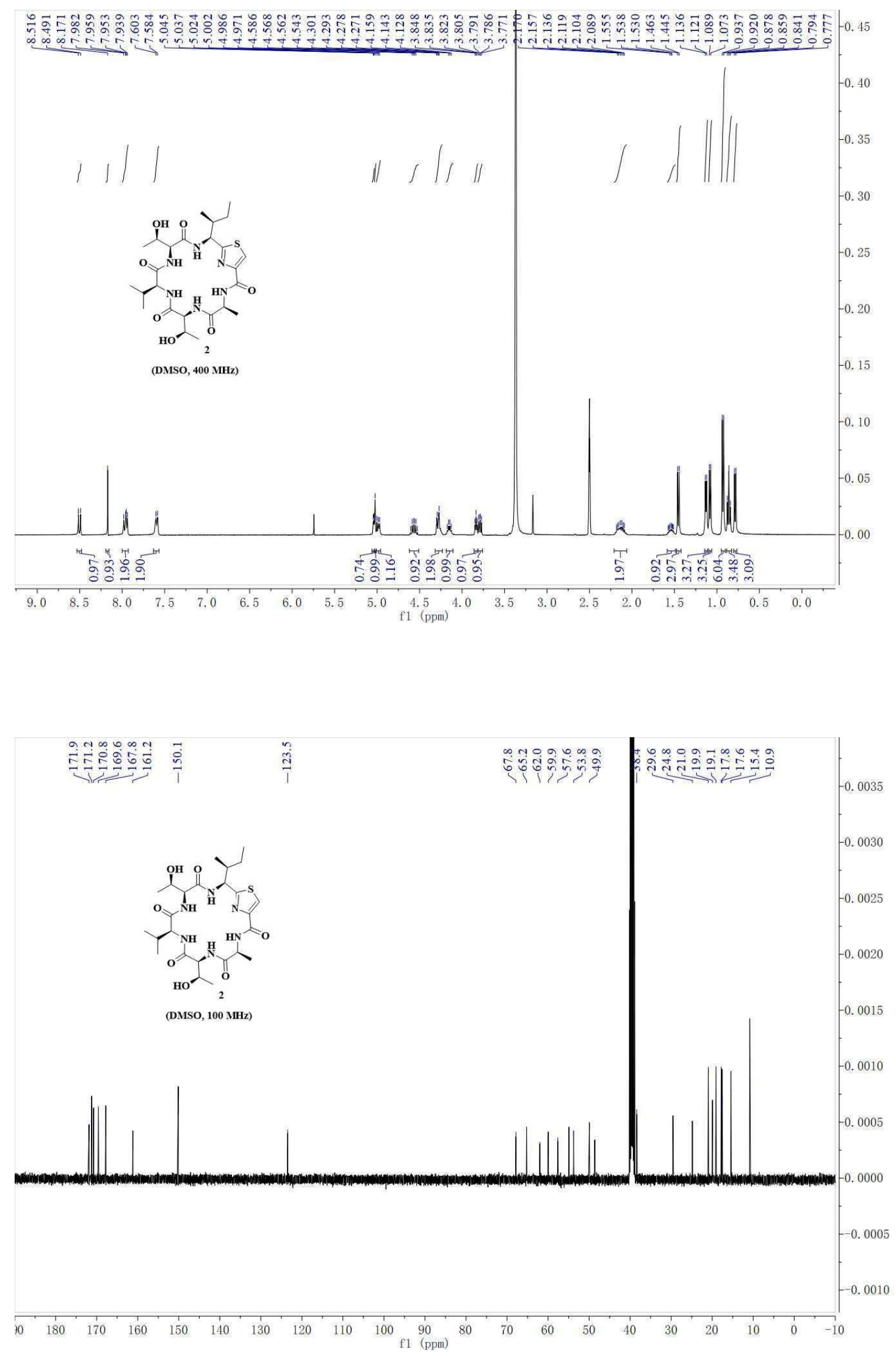


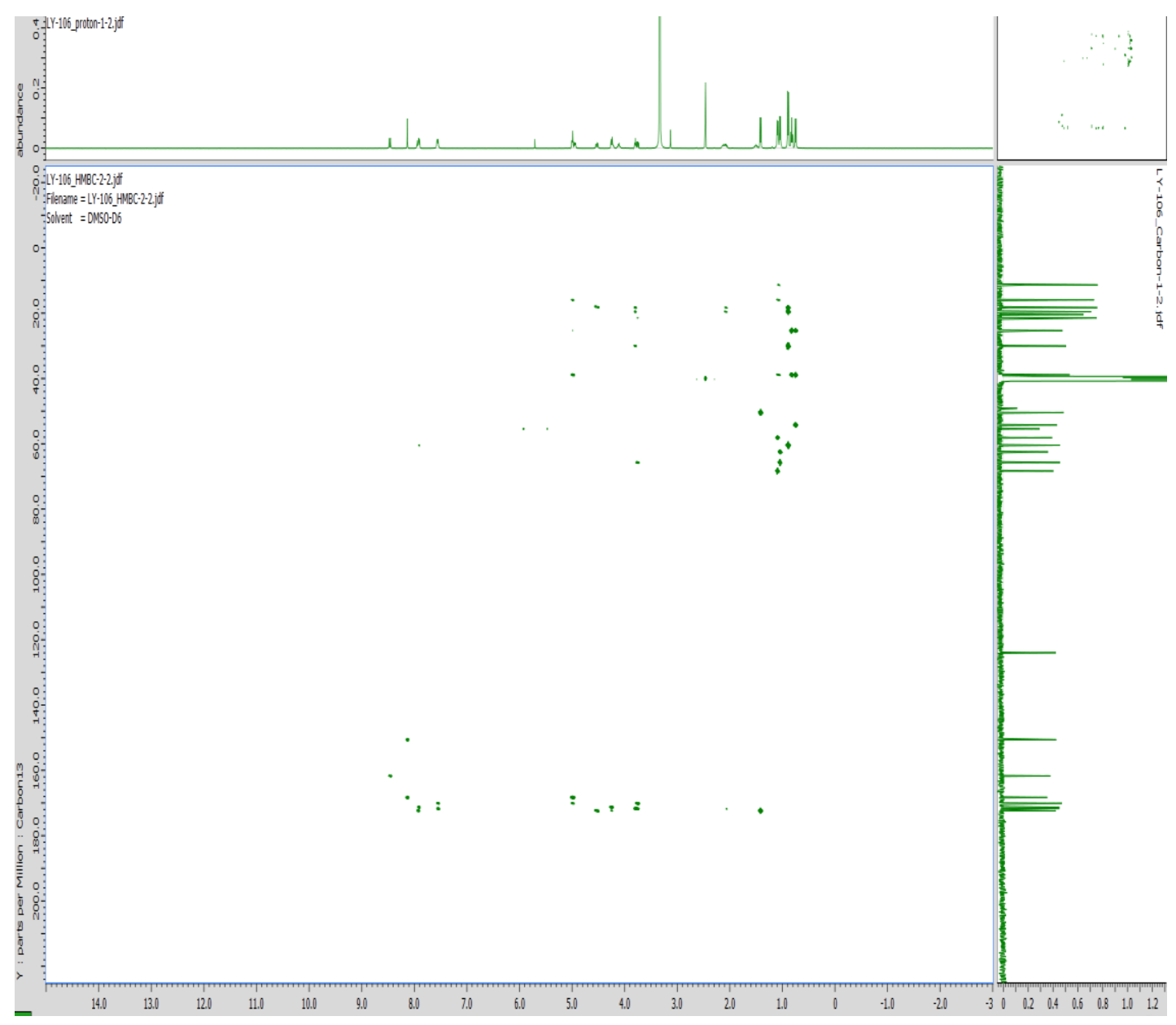

